\title{
Stability Properties and the KMS Condition
}

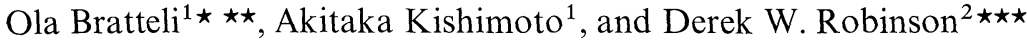 \\ ${ }^{1}$ Centre de Physique Théorique II, CNRS, F-13274 Marseille Cedex 2, France \\ 2 Département de Physique, Université d'Aix-Marseille II, Luminy, F-13274 Marseille, and Centre de \\ Physique Théorique II, CNRS, F-13274 Marseille Cedex 2, France
}

\begin{abstract}
First we derive stability properties of KMS states and subsequently we derive the KMS condition from stability properties. New results include a convergent perturbation expansion for perturbed KMS states in terms of appropriate truncated functions and stability properties of ground states. Finally we extend the results of Haag, Kastler, Trych-Pohlmeyer by proving that stable states of $L^{1}$-asymptotically abelian systems which satisfy a weak three point cluster property are automatically KMS states. This last theorem gives an almost complete characterization of KMS states, of $L^{1}$-asymptotic abelian systems, by stability and cluster properties (a slight discrepancy can occur for infinite temperature states).
\end{abstract}

\section{Introduction}

Let $(\mathfrak{H}, \tau)$ denote a $C^{*_{-}}$(or $W^{*_{-}}$) dynamical system composed of a $C^{*_{-}}$(or $W^{*_{-}}$) algebra $\mathfrak{A}$ and a strongly continuous ( $\sigma$-weakly continuous) one-parameter group of *-automorphisms $\tau$ of $\mathfrak{Q}$. If $\delta$ is the generator of $\tau$ and for $P=P^{*} \in \mathfrak{A}$ one defines $\delta_{P}$ by $\delta_{P}(A)=i[P, A]$ then one can introduce the automorphism group $\tau^{P}$ generated by $\delta+\delta_{P}$ and compare the structures associated with $(\mathfrak{H}, \tau)$ and the perturbed system $\left(\mathfrak{A}, \tau^{P}\right)$.

There are two approaches to this study. The first is a "time-independent" perturbation theory pioneered by Araki [2,3], and relying on results of Connes [8]. This method is designed for comparison of $\tau$-KMS states and $\tau^{p}$-KMS states, i.e. modular states over $(\mathfrak{H}, \tau)$, and $\left(\mathfrak{H}, \tau^{P}\right)$, respectively. The second approach is a "time-dependent" perturbation theory introduced by Robinson [16]. This second method allows comparison of more general states for systems satisfying suitable ergodicity hypotheses, e.g. conditions of asymptotic abelianness.

* Supported during this research by the Norwegian Research Council for Science and Humanities

$\star \star$ Present address: Matematisk Institutt, Avd. A, Universitetet i Oslo, Blindern, Oslo 3, Norway $\star \star \star$ Present address: Department of Mathematics, University of New South Wales, P.O. Box 1, Kensington, NSW 2033, Australia 
The primary object of these investigations is the set of "stable structures" and various notions of stability are possible. The method of Araki and Connes establishes that there is a one-to-one correspondence between $(\tau, \beta)$-KMS states and $\left(\tau^{P}, \beta\right)$-KMS states for all $\beta \in \mathbb{R}$ and that every extremal $\left(\tau^{P}, \beta\right)$-KMS state is a vector state of an extremal $(\tau, \beta)$-KMS state. This normality is a natural form of stability. On the other hand Robinson [16] remarked that a $\tau^{P}$-invariant state $\omega^{P}$ evolves to a unique $\tau$-invariant state $\omega$, i.e.

$$
\omega(A)=\lim _{|t| \rightarrow \infty} \omega^{P}\left(\tau_{t}(A)\right)
$$

for all $A \in \mathfrak{U}$, if, and only if,

$$
\lim _{T \rightarrow \infty} \int_{-T}^{T} d t \omega^{P}\left(\left[P, \tau_{t}(A)\right]\right)=0
$$

for all $A \in \mathfrak{A}$. Now if $\omega^{P}$ is contained in a family of states $\omega^{\lambda P}, 0<\lambda \leqq 1$, for which $\omega^{\lambda P} \rightarrow \omega$ in a suitable sense as $\lambda \rightarrow 0$ then one formally obtains the "stability condition"

$$
\lim _{T \rightarrow \infty} \int_{-T}^{T} d t \omega\left(\left[P, \tau_{t}(A)\right]=0 .\right.
$$

This form of stability was introduced by Haag, Kastler and Trych-Pohlmeyer [10]. These authors proved that if the condition is valid for a dense set of $P, A \in \mathfrak{A}$, and if $(\mathfrak{A}, \tau, \omega)$ satisfy certain ergodicity assumptions, then $\omega$ is automatically a $(\tau, \beta)$-KMS state for some $\beta \in \mathbb{R} \cup\{ \pm \infty\}$ (see also $[6,12,13]$ ). This striking result showed that stability and the KMS condition are to a large extent equivalent.

Since these early works there have been two types of investigation of stability. The first derives stability conditions for special states, e.g. KMS states or ground states, and the second aims at deducing the KMS condition from stability and ergodicity. Although the initial investigations were quantum-mechanical subsequent studies have examined the classical case $[1,14]$. We give various results of the first kind in Section 2, and a general theorem of the second kind, partially based on a technique of [11], in Section 3.

\section{Stability Properties}

Let $\beta \in \mathbb{R}^{1}$. A state $\omega$ over the $C^{*_{-}}\left(W^{*_{-}}\right)$dynamical system $(\mathfrak{U}, \tau)$ is defined to be a $(\tau, \beta)$-KMS state if

$$
\omega\left(A \tau_{i \beta}(B)\right)=\omega(B A)
$$

for all $A, B$ in a norm ( $\sigma$-weakly) dense $\tau$-invariant *-subalgebra of the $*_{\text {-algebra }}$ $\mathfrak{H}_{\tau}$ of entire analytic elements for $\tau$ (and $\omega$ is normal in the $W^{*}$ case). It follows that $\omega$ is $\tau$-invariant when $\beta \neq 0$, and we let $\left(\mathscr{H}_{\omega}, \Pi_{\omega}, U_{\omega}, \Omega_{\omega}\right)$ denote the covariant cyclic representation generated by $\omega$. Moreover $\Omega_{\omega}$ is separating for $\Pi_{\omega}(\mathfrak{U})^{\prime \prime}$ and $\tau$ is the modular group associated with the pair $\left(\Pi_{\omega}(\mathfrak{U})^{\prime \prime}, \Omega_{\omega}\right)$ if $\beta=-1$. For any pair

In the arguments of the rest of this section it is implicitly assumed that $\beta>0$. The case $\beta<0$ can be treated analogously, while Theorems 1 and 2 are trivial in the case $\beta=0$ 
$A, B \in \Pi_{\omega}(\mathfrak{H})^{\prime \prime}$ there exists a complex function $F_{A, B}$ which is analytic on $\mathscr{D}_{\beta}$ $=\{z ; \operatorname{Im} z \in(0, \beta)\}$, bounded and continuous on $\overline{\mathscr{D}}_{\beta}$, and satisfies

$$
\begin{aligned}
F_{A, B}(t) & =\left(\Omega_{\omega}, A U_{\omega}(t) B \Omega_{\omega}\right) \\
F_{A, B}(t+i \beta) & =\left(\Omega_{\omega}, B U_{\omega}(-t) A \Omega_{\omega}\right) .
\end{aligned}
$$

If $A, B \in \mathfrak{A}_{\tau}$ then $F_{A, B}(z)=\omega\left(A \tau_{z}(B)\right)$ and by "abus de notation" we will consistently write

$$
F_{A, B}(z)=\omega\left(A \tau_{z}(B)\right) .
$$

These results extend to several variables $[2,3]$. If

$$
\mathscr{D}_{\beta}^{(n)}=\left\{z=\left(z_{1}, \ldots, z_{n}\right), \operatorname{Im} z_{1} \in(0, \beta), \operatorname{Im} z_{j} \in\left(0, \operatorname{Im} z_{j-1}\right), j=z, \ldots, n\right\}
$$

and $A, B_{1}, \ldots, B_{n} \in \Pi_{\omega}(\mathfrak{Q})^{\prime \prime}$ then the function

$$
F_{A,\left\{B_{3}\right\}}(t)=\left(\Omega_{\omega}, A \hat{\tau}_{t_{1}}\left(B_{1}\right) \ldots \hat{t}_{t_{n}}\left(B_{n}\right) \Omega_{\omega}\right),
$$

where $\hat{\tau}_{t_{j}}\left(B_{j}\right)=U_{\omega}\left(t_{j}\right) B_{j} U_{\omega}\left(t_{j}\right)^{*}$, has a holomorphic extension to the tube $\mathscr{D}_{\beta}^{(n)}$ which is continuous and uniformly bounded on $\overline{\mathscr{D}_{\beta}^{(n)}}$. We also write

$$
F_{A,\left\{B_{j}\right\}}(z)=\omega\left(A \tau_{z_{1}}\left(B_{1}\right) \ldots \tau_{z_{n}}\left(B_{n}\right)\right)
$$

for this function and this is consistent if $B_{1}, \ldots, B_{n} \in \mathfrak{A}_{\tau}$. The bounds

$$
\sup _{z \in \mathscr{Q}(\underline{\beta})}\left|F_{A,\left\{B_{i}\right\}}(z)\right| \leqq\|A\| \prod_{j=1}^{n}\left\|B_{j}\right\|
$$

are valid.

Next define the perturbed group $\tau^{P}$ as the group with generator $\delta+\delta_{P}$ where $\delta$ is the generator of $\tau$ and $\delta_{P}(A)=i[P, A]$ for $P=P^{*} \in \mathfrak{A}$ and all $A \in \mathfrak{A}$. Thus $\tau^{P}$ is the unique solution of the norm ( $\sigma$-weak) differential equation

$$
\frac{d \tau_{t}^{P}(A)}{d t}=\tau_{t}^{P}\left(\delta(A)+\delta_{P}(A)\right)
$$

for $A \in D(\delta)$. This can be solved by integration and iteration and one finds

$$
\tau_{t}^{P}(A)=\tau_{t}(A)+\sum_{n \geqq 1} i^{n} \int_{0}^{t} d t_{1} \int_{0}^{t_{1}} d t_{2} \ldots \int_{0}^{t_{n-1}} d t_{n}\left[\tau_{t_{n}}(P)\left[\ldots\left[\tau_{t_{1}}(P), \tau_{t}(A)\right]\right]\right] .
$$

This is the original definition given by Robinson [16]. There is an alternative formulation of $\tau_{P}$ which was independently derived by Araki [2], Connes [8], and Robinson [16]. First one defines $\Gamma_{t}^{P} \in \mathfrak{A}$ as the unique solution of $\Gamma_{0}^{P}=\mathbb{1}$ and

$$
\frac{\mathrm{d} \Gamma_{t}^{P}}{d t}=i \Gamma_{t}^{P} \tau_{t}(P)
$$

Thus

$$
\Gamma_{t}^{P}=\mathbb{1}+\sum_{n \geqq 1} i^{n} \int_{0}^{t} d t_{1} \int_{0}^{t_{1}} d t_{2} \ldots \int_{0}^{t_{n-1}} d t_{n} \tau_{t_{n}}(P) \ldots \tau_{t_{1}}(P) .
$$


One then finds that $\Gamma_{t}^{P}$ is unitary, it satisfies the co-cycle relation

$$
\Gamma_{t+s}^{P}=\Gamma_{t}^{P} \tau_{t}\left(\Gamma_{s}^{P}\right)
$$

and

$$
\tau_{t}^{P}(\mathrm{~A})=\Gamma_{t}^{P} \tau_{t}(\mathrm{~A}) \Gamma_{t}^{P *} .
$$

If $\omega$ is a $(\tau, \beta)$-KMS state then $t \mapsto \omega\left(A \Gamma_{t}^{P}\right)$ has an analytic extension to the strip $\mathscr{D}_{\beta}$ which can be constructed via the functions $F_{A, P}(z)$ and the perturbation series. One has in particular

$$
\omega\left(A \Gamma_{i \alpha}^{P}\right)=\omega(A)+\sum_{n \geqq 1}(-1)^{n} \int_{0}^{\alpha} d s_{1} \int_{0}^{s_{1}} d s_{2} \ldots \int_{0}^{s_{n-1}} d s_{n} \omega\left(A \tau_{i s_{1}}(P) \ldots \tau_{i s_{n}}(P)\right)
$$

for $\alpha \in[0, \beta]$. The bounds on $F_{A, P}$ then establish that $\lambda \in \mathbb{R} \mapsto \omega\left(A \Gamma_{i \beta}^{\lambda P}\right)$ is an entire analytic function. A key result of Araki is that $\omega\left(\Gamma_{i \beta}^{P}\right)>0$ and the state $\omega^{P}$ defined by

$$
\omega^{P}(A)=\omega\left(A \Gamma_{i \beta}^{P}\right) \omega\left(\Gamma_{i \beta}^{P}\right)
$$

is a $\left(\tau^{P}, \beta\right)$-KMS state. Moreover $\omega$ is an extremal $(\tau, \beta)$-KMS state if, and only if, $\omega^{P}$ is an extremal $\left(\tau^{P}, \beta\right)$-KMS state. A simple proof of this is given in [17].

It follows from the above that $\lambda \mapsto \omega^{\lambda P}(A)$ is analytic in a neighbourhood of the origin and our first result concerns the coefficients of the Taylor series expansion of this function and its radius of convergence. The coefficients are identifiable as integrals of truncated functions and we must first recall the definition of such functions.

Let $\mathfrak{I}$ denote an arbitrary index set and $F$ a function from the non-empty ordered finite subsets of $\mathfrak{I}$ to the complex numbers. The truncation $F_{T}$ of $F$ is defined recursively by

$$
F(I)=\sum_{\mathscr{P}_{I}} \prod_{J \in \mathscr{P}_{I}} F_{T}(J),
$$

where the sum is over all partitions $\mathscr{P}_{I}$ of the finite set $I$, and the elements of each $J \in \mathscr{P}_{I}$ retain the order of $I$. If $\alpha$ is any point in $I$ one then has

$$
F(I)=\sum_{J \subset I, J \ni \alpha} F_{T}(J) F(I \backslash J)
$$

This follows directly from the recursion relations by noting that the coefficient of an arbitrary term $F_{T}(J)$ in these relations is given by

$$
\sum_{\mathscr{P}_{I / J}} \prod_{K \in \mathscr{P}_{I / J}} F_{T}(K)=F(I \backslash J) .
$$

These definitions can now be applied to a state $\omega$ and a set $A_{i}$ of elements of $\mathfrak{A}$ to define truncations $\omega_{T}\left(A_{1} ; A_{2} ; \ldots ; A_{n}\right)$ of $\omega\left(A_{1} A_{2} \ldots A_{n}\right)$.

Theorem 1. Let $(\mathfrak{A}, \tau)$ be a $C^{*}$-, or $W^{*}$-, dynamical system and $\omega$ a $(\tau, \beta)$-KMS state over $\mathfrak{A}$.

It follows that for each $A \in \mathfrak{U}$ and $P=P^{*} \in \mathfrak{U}$ the truncated function

$$
F_{A}\left(t_{1}, \ldots, t_{n}\right)=\omega_{T}\left(A ; \tau_{t_{n}}(P) ; \ldots ; \tau_{t_{1}}(P)\right)
$$


is the boundary value of a function $F_{A}(z)\left(=\omega_{T}\left(A ; \tau_{z^{n}}(P) ; \ldots ; \tau_{z_{1}}(P)\right)\right.$ which is holomorphic in the tube $\mathscr{D}_{\beta}^{(n)}$, continuous and uniformly bounded on its closure $\overline{\mathscr{D}_{\beta}^{n}}$, and

$$
\sup _{z \in \mathscr{D}(n)}\left|F_{A}(z)\right| \leqq 2^{n} n !\|P\|^{n}\|A\| .
$$

Moreover if $2\|P\|<1$ the perturbed $\left(\tau^{P}, \beta\right)$-KMS state $\omega^{P}$ given by

$$
\omega^{P}(A)=\omega\left(A \Gamma_{i \beta}^{P}\right) / \omega\left(\Gamma_{i \beta}^{P}\right)
$$

is determined by the uniformly convergent series

$$
\omega^{P}(A)=\omega(A)+\sum_{n \geqq 1}(-1)^{n} \int_{0}^{\beta} d s_{1} \ldots \int_{0}^{s_{n}-1} d s_{n} \omega_{T}\left(A ; \tau_{i s_{n}}(P) ; \ldots ; \tau_{i s_{1}}(P)\right) .
$$

Proof. The truncated functions are finite linear combinations of the non-truncated functions and these latter are holomorphic in $\mathscr{D}_{\beta}^{(n)}$ etc. If we define $F_{A}$ as the appropriate combination of these holomorphic functions we obtain the first statement.

To derive the bound on $F_{A}$ we first note that $\omega_{T}$ satisfies the KMS condition

$$
\begin{aligned}
& \omega_{T}\left(A ; \tau_{t_{n}}(P) ; \ldots ; \tau_{t_{j+1}}(P) ; \tau_{t_{j}+i \beta}(\mathrm{P}) ; \ldots ; \tau_{t_{1}+i \beta}(P)\right) \\
& \quad=\omega_{T}\left(\tau_{t_{j}}(P) ; \ldots ; \tau_{t_{1}}(P) ; A ; \tau_{t_{n}}(P) ; \ldots ; \tau_{t_{j+1}}(P)\right) .
\end{aligned}
$$

This follows from the definition of $\omega_{T}$ and the KMS condition for $\omega$. But the Phragmen-Lindelöf theorem implies that $F_{A}$ attains its supremum on one of the edges $\operatorname{Im} z_{1}=\operatorname{Im} z_{2}=\ldots=\operatorname{Im} z_{j}=\beta, \operatorname{Im} z_{j+1}=\operatorname{Im} z_{j+2}=\ldots=\operatorname{Im} z_{n}=0$. Hence

$$
\sup _{z \in \mathscr{Q} \mathcal{X}_{\beta}^{(n)}}\left|F_{A}(z)\right| \leqq \sup _{1 \leqq j \leqq n} \sup _{t \in \mathbb{R}^{n}}\left|\omega_{T}\left(\tau_{t_{j}}(P) ; \ldots ; \tau_{t_{1}}(P) ; A ; \ldots ; \tau_{t_{j-1}}(P)\right)\right| .
$$

But a function $F$ and its truncations $F_{T}$ are related by

$$
F(I)=F_{T}(I)+\sum_{\substack{\alpha \in J_{\subsetneq} I \\ \neq}} F_{T}(J) F(I \backslash J) .
$$

Thus if $|F(I)| \leqq 1$, and $\left|F_{T}(I)\right|<2^{|I|-1}(|I|-1)^{1}$ for $|I| \leqq n$ where $n>2(|I|$ denotes the number of points in $I$, then for $|I|=n+1$

$$
\begin{aligned}
\left|F_{T}(I)\right| & \leqq 1+\sum_{m=1}^{n}{ }^{n} C_{m-1} 2^{m-1}(m-1) ! \\
& =2^{n} n !\left\{\left(2^{n} n !\right)^{-1}+\sum_{m=1}^{n} \frac{1}{(n-m+1) !} \frac{1}{2^{n-m+1}}\right\} \\
& \leqq 2^{n} n !\left\{\frac{1}{8}+e^{1 / 2}-1\right\}<2^{n} n !
\end{aligned}
$$

We can apply this result to $\omega_{T}\left(A_{1} ; A_{2} ; \ldots ; A_{n}\right)$ with $\left\|A_{i}\right\| \leqq 1$. One has $\left|\omega\left(A_{1}, A_{2} \ldots A_{n}\right)\right| \leqq 1$ and $\left|\omega_{T}\left(A_{i}\right)\right| \leqq 1,\left|\omega_{T}\left(A_{i} ; A_{j}\right)\right| \leqq 2$ and hence

$$
\left|\omega_{T}\left(A_{1} ; A_{2} ; \ldots ; A_{n}\right)\right| \leqq 2^{n-1}(n-1) !
$$

for all $n \geqq 1$ by induction. 
Combination of these results immediately yields

$\sup _{z \in \mathscr{D}_{\beta}^{(n)}}\left|F_{A}(z)\right| \leqq 2^{n} n !\|P\|^{n}\|A\|$

Next suppose $\lambda \in \mathbb{R} \rightarrow \omega^{\lambda P}$ has the power series expansion

$$
\omega^{\lambda P}(A)=\sum_{n \geqq 0} \lambda^{n} \hat{\omega}_{n}^{P}(A) .
$$

Define $\omega_{n}^{P}(A)$ by $\omega_{0}^{P}(A)=\omega(A)$ and

$$
\omega_{n}^{P}(A)=(-1)^{n} \int_{0}^{\beta} d s_{1} \ldots \int_{0}^{s_{n-1}} d s_{n} \omega\left(A \tau_{i s_{n}}(P) \ldots \tau_{i s_{1}}(P)\right) .
$$

Hence

$$
\omega^{\lambda P}(A)=\left(\sum_{n \geqq 0} \omega_{n}^{\lambda P}(A)\right) /\left(\sum_{n \geqq 0} \omega_{n}^{\lambda P}(\mathbb{1})\right) .
$$

Multiplication by the denominator on the right and term by term comparison of power series then gives

$$
\omega_{n}^{P}(A)=\hat{\omega}_{n}^{P}(A)+\sum_{r=1}^{n} \hat{\omega}_{n-r}^{P}(A) \omega_{r}^{P}(\mathbb{1}) .
$$

In particular $\hat{\omega}_{0}^{P}(A)=\omega(A)$ and

$$
\begin{aligned}
\hat{\omega}_{1}^{P}(A) & =\omega^{P}(A)-\omega(A) \omega_{1}^{P}(\mathbb{1}) \\
& =-\int_{0}^{\beta} d s \omega_{T}\left(A \tau_{i s}(P)\right) .
\end{aligned}
$$

Now for $I=\left\{i_{m}, \ldots, i_{1}\right\}$ adopt the notation

$$
\begin{aligned}
& \left.\omega(A ; I)=\omega\left(A_{i i_{i_{1}}}(P) \ldots \tau_{i s_{i_{m}}} P\right)\right) \\
& \omega_{T}(A ; I)+\omega\left(A ; \tau_{i s_{i_{1}}}(P) ; \ldots ; \tau_{i s_{s_{m}}}(P)\right)
\end{aligned}
$$

and assume that

$$
\hat{\omega}_{r}^{P}(A)=(-1) \int_{0}^{\beta} d s_{1} \ldots \int_{0}^{s_{r-1}} d s_{r} \omega_{T}(A ;\{1,2, \ldots, r\})
$$

for $r \leqq n-1$. But then

$$
\begin{aligned}
\omega_{n}^{P}(\mathrm{~A})= & \hat{\omega}_{n}^{P}(A)+(-1)^{n} \sum_{r=0}^{n-1} \int_{0}^{\beta} d s_{n-r+1} \ldots \int_{0}^{s_{n-1}} d s_{n} \omega_{T}(A ;\{n-r+1, \ldots, n\}) \\
& \cdot \int_{0}^{\beta} d s_{1} \ldots \int_{0}^{s_{n-r-1}} d s_{n-r} \omega(\mathbb{1} ;\{, \ldots, n-r\})
\end{aligned}
$$

by $(*)$ and the definition of $\omega_{r}^{P}(\mathbb{1})$. By change of integration variable this gives

$$
\omega_{n}^{P}(A)=\hat{\omega}_{n}^{P}(A)+(-1)^{n} \int_{0}^{\beta} d s_{1} \ldots \int_{0}^{s_{n}-1} d s_{n} \sum_{J \subsetneq I} \omega_{T}(A ; J) \omega(\mathbb{1} ; I \backslash J),
$$


where $I=\{1, \ldots, n\}$ and the sum is over the strict subsets of $I$. But the relation between a function and its truncations gives

$$
\sum_{J \subsetneq I} \omega_{T}(A ; J) \omega(\mathbb{1} ; I \backslash J)=\omega(A ; I)-\omega_{T}(A ; I)
$$

and hence

$$
\begin{aligned}
\hat{\omega}_{n}^{P}(A) & =\omega_{n}^{P}(A)-(-1)^{n} \int_{0}^{\beta} d s_{1} \ldots \int_{0}^{s_{n-1}} d s_{n}\left\{\omega(A ; I)-\omega_{T}(A ; I)\right\} \\
& =(-1)^{n} \int_{0}^{\beta} d s_{1} \ldots \int_{0}^{s_{n-1}} d s_{n} \omega_{T}(A ; I) .
\end{aligned}
$$

This establishes the correct identification of the perturbation series for $\omega^{P}$ by induction.

Next consider the orbit of $\omega$ under the evolution $\tau^{P}$. The time dependent formalism developed in [16] indicates that under certain general ergodicity hypotheses $\omega$ evolves into a $\left(\tau^{P}, \beta\right)$-KMS state. If $\omega$ is extremal, it is natural to define the system as stable if the evolved state is the unique $\left(\tau^{P}, \beta\right)$-KMS vector state $\omega^{P}$ of $\omega$. Explicitly this definition of stability requires that

$$
\omega^{P}(A)=\lim _{t \rightarrow \pm \infty} \omega\left(\tau_{t}^{P}(A)\right) .
$$

But it then follows from the $\tau$-invariance of $\omega$ and the iterative form for $\tau^{P}$ that

$$
\begin{aligned}
\omega^{P}(A) & =\lim _{t \rightarrow \pm \infty} \omega\left(\tau_{-t} \tau_{t}^{P}(A)\right) \\
& =\omega(A)+\lim _{t \rightarrow \pm \infty} \sum_{n \geqq 1}(-i)^{n} \int_{0}^{t} d t_{1} \ldots \int_{t_{n-1}}^{t} d t_{n} \omega\left(\left[\tau_{t_{n}}(P)\left[\ldots\left[\tau_{t_{1}}(P), A\right] \ldots\right]\right]\right) .
\end{aligned}
$$

Thus one obtains a series for $\omega^{P}$ which is seemingly different to that of Theorem 1 . The identity of these series, term-by-term yields a set of stability conditions which we next derive for strongly clustering $\omega$.

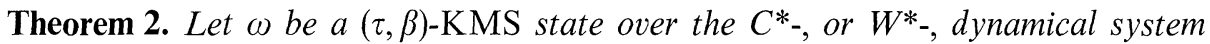
$(\mathfrak{U}, \tau)$ and assume that $\omega$ is strongly clustering, i.e.

$$
\lim _{t \rightarrow \pm \infty} \omega\left(A \tau_{t}(B)\right)=\omega(A) \omega(B)
$$

for all $A, B \in \mathfrak{U}$.

It follows that

$$
\begin{aligned}
& \lim _{T_{1} \rightarrow \pm \infty} \ldots \lim _{T_{n} \rightarrow \pm \infty} i^{n} \int_{0}^{T_{1}} d t_{1} \int_{t_{1}}^{T_{2}} d t_{2} \ldots \int_{t_{n-1}}^{T_{n}} d t_{n} \omega\left(\left[\tau_{t_{n}}(P),\left[\ldots,\left[\tau_{t_{1}}(P), A\right] \ldots\right]\right]\right) \\
& =\int_{0}^{\beta} d s_{1} \ldots \int_{0}^{s_{n-1}} d s_{n} \omega_{T}\left(A ; \tau_{i s_{n}}(\mathrm{P}) ; \ldots ; \tau_{i s_{1}}(P)\right)
\end{aligned}
$$

for all $A, P \in \mathfrak{A}$ and in particular

(*) $\quad \lim _{T \rightarrow \infty} \int_{-T}^{T} d t \omega\left(\left[A, \tau_{t}(P)\right]\right)=0$

for all $A, P \in \mathfrak{U}$. 
Proof. Let $P \in \mathfrak{U}_{\tau}$ and introduce $P\left(s_{1}, \ldots, s_{j}\right)=\tau_{i s_{j}}(P) \ldots \tau_{i s_{1}}(P)$. We first argue that

$$
\begin{aligned}
& \int_{t_{j}}^{T_{j+1}} d t_{j+1} \int_{0}^{\beta} d s_{1} \ldots \int_{0}^{s_{j-1}} d s_{j} \omega\left(\left[\tau_{t_{j+1}}(P), B\right] \tau_{t_{j+1}}\left(P\left(s_{1}, \ldots, s_{j}\right)\right)\right) \\
& =-i \int_{0}^{\beta} d s_{1} \ldots \int_{0}^{s_{j}} d s_{j+1} \omega\left(B \tau_{t_{j}}\left(P\left(s_{1}, \ldots, s_{j+1}\right)\right)-B \tau_{T_{j+1}}\left(P\left(s_{1}, \ldots, s_{j+1}\right)\right)\right) .
\end{aligned}
$$

This follows from the KMS condition and contour integration. The KMS condition allows one to re-express the left hand side as the difference of two terms $L_{1}$ and $L_{2}$, where

$$
\begin{aligned}
& L_{1}=\int_{t_{j}}^{T_{j+1}} d t_{j+1} \int_{0}^{\beta} d s_{1} \ldots \int_{0}^{s_{j-1}} d s_{j} \omega\left(B \tau_{t_{j+1}}\left(P\left(\beta, s_{1}, \ldots, s_{j}\right)\right)\right), \\
& L_{2}=\int_{t_{j}}^{T_{j+1}} d t_{j+1} \int_{0}^{\beta} d s_{1} \ldots \int_{0}^{s_{j-1}} d s_{j} \omega\left(B \tau_{t_{j+1}}\left(P\left(s_{1}, \ldots, s_{j}, 0\right)\right)\right) .
\end{aligned}
$$

Next by a change of variable $s_{k}^{\prime}=s_{k+1}-s_{k}+\beta ; k=1, \ldots, j-1$ and $s_{j}^{\prime}=\beta-s_{1}$ and a subsequent shift of the contour of integration one finds

$$
\begin{aligned}
L_{2}= & \int_{t_{j}}^{T_{j+1}} d t_{j+1} \int_{0}^{\beta} d s_{1} \ldots \int_{0}^{s_{j-1}} d s_{j} \omega\left(B \tau_{t_{j+1}}\left(P\left(\beta-s_{j} ; s_{1}-s_{j}, \ldots, s_{j-1}-s_{j}, 0\right)\right)\right) \\
= & i \int_{0}^{\beta} d s_{1} \ldots \int_{0}^{s_{j}} d s_{j+1}\left\{\omega\left(B \tau_{t_{j}}\left(P\left(\beta-s_{j}+s_{j+1}, \ldots, s_{j-1}-s_{j}+s_{j+1}, s_{j+1}\right)\right)\right)\right. \\
& \left.-\omega\left(B \tau_{T_{j+1}}\left(P\left(\beta-s_{j}+s_{j+1}, \ldots, s_{j-1}-s_{j}+s_{j+1}, s_{j+1}\right)\right)\right)\right\} \\
& +L_{1} .
\end{aligned}
$$

Another change of variable gives the desired identification of $L_{1}-L_{2}$. A similar identity is valid for a general $P \in \mathfrak{A}$ by approximation with analytic elements.

Next define $C$ and $X$ by

$$
\begin{aligned}
C\left(t_{1}, \ldots, t_{n}\right) & =\left[\tau_{t_{n}}(P),\left[\ldots,\left[\tau_{t_{1}}(P), A\right] \ldots\right]\right. \\
X\left(T_{1}, \ldots, T_{n}\right) & =\int_{0}^{T_{1}} d t_{1} \int_{t_{1}}^{T_{2}} d t_{2} \ldots \int_{t_{n-1}}^{T_{n}} d t_{n} \omega\left(C\left(t_{1}, \ldots, t_{n}\right)\right) .
\end{aligned}
$$

Application of the above identity and strong clustering gives

$$
\begin{aligned}
\lim _{T_{1} \ldots T_{n} \rightarrow \pm \infty} X\left(T_{1}, \ldots, T_{n}\right)= & \sum_{j=1}^{n-1}-(-i)^{n-j} \lim _{T_{1} \ldots T_{j} \rightarrow \pm \infty} \int_{0}^{T_{1}} d t_{1} \ldots \int_{t_{j-1}}^{T_{j}} d t_{j} \omega\left(C\left(t_{1} \ldots t_{j}\right)\right) \\
& \cdot \int_{0}^{\beta} d s_{1} \ldots \int_{0}^{s_{n-1}} d s_{n-j} \omega\left(P\left(s_{1}, \ldots, s_{n-j}\right)\right) \\
& +(-i)^{n} \int_{0}^{\beta} d s_{1} \ldots \int_{0}^{s_{n-1}} d s_{n} \omega\left(A P\left(s_{1}, \ldots, s_{n}\right)\right)
\end{aligned}
$$

for $n \geqq 2$ and

$$
\lim _{T_{1} \rightarrow \pm \infty} X\left(T_{1}\right)=-i \int_{0}^{\beta} d s_{1}\left\{\omega\left(A \tau_{i s_{1}}(P)\right)-\omega(A) \omega(P)\right\} .
$$


The proof is concluded by induction. The last equation establishes the case $n=1$. Suppose the result is true for $j=1, \ldots, n-1$, then the above identity gives

$$
\begin{aligned}
\lim _{T_{1} \ldots T_{n} \rightarrow \pm \infty} X\left(T_{1}, \ldots, T_{n}\right) \\
=-(-i)^{n} \sum_{j=1}^{n-1} \int_{0}^{\beta} d s_{1} \ldots \int_{0}^{s_{n-j-1}} d s_{n-j} \omega\left(\tau_{i s_{n-j}}(P) \ldots \tau_{i s_{1}}(P)\right) \\
\quad \cdot \int_{0}^{\beta} d s_{n-j+1} \ldots \int_{0}^{s_{n-1}} d s_{n} \omega_{T}\left(A ; \ldots ; \tau_{i s_{n-i+1}}(P)\right) \\
+(-i)^{n} \int_{0}^{\beta} d s_{1} \ldots \int_{0}^{s_{n-1}} d s_{n} \omega\left(A \tau_{i s_{n}}(P) \ldots \tau_{i s_{1}}(P)\right) \\
=(-i)^{n} \int_{0}^{\beta} d s_{1} \ldots \int_{0}^{s_{n-1}} d s_{n} \omega_{T}\left(A ; \tau_{i s_{n}}(P) ; \ldots ; \tau_{i s_{1}}(P)\right),
\end{aligned}
$$

where we have changed the integration variables and used the general relation between a function and its truncations as in the conclusion of the proof of Theorem 1. This completes the induction.

The last statement of the theorem follows by subtracting the two limits $T \rightarrow \pm \infty$ in the case $n=1$.

Next we examine stability and instability of ground states, and ceiling states. There are various equivalent definitions of such states. We define $\omega$ to be a $\tau$ ground state of $\mathfrak{A}$ if

$$
-i \omega\left(A^{*} \delta(A)\right) \geqq 0
$$

for all $A \in D(\delta)$ where $\delta$ is the generator of $\tau$. Similarly $\omega$ is called a $\tau$-ceiling state if

$$
i \omega\left(A^{*} \delta(A)\right) \geqq 0 \text {. }
$$

These states are also called $(\tau,+\infty)$-KMS states, and $(\tau,-\infty)$-KMS states, respectively. This is motivated by the fact that if a sequence $\omega_{n}$ of $\left(\tau, \beta_{n}\right)$-KMS states converges weak* to a state $\omega$ as $\beta_{n} \rightarrow \infty\left(\beta_{n} \rightarrow-\infty\right)$ then $\omega$ is a $\tau$-ground state ( $\tau$-ceiling state). This convergence property is an easy consequence of an infinitesimal generator characterization of KMS states given by Sewell [18], see [7].

Alternatively, and equivalently, $\omega$ is a $\tau$-ground state if $\omega$ is $\tau$-invariant and if the corresponding unitary representation $U_{\omega}(t)=e^{i t H_{\omega}}$ given by

$$
U_{\omega}(t) \Pi_{\omega}(A) \Omega_{\omega}=\Pi_{\omega}\left(\tau_{t}(A)\right) \Omega_{\omega}
$$

has the property $H_{\omega} \geqq 0$. If this is the situation then $U_{\omega}(t) \in \Pi_{\omega}(\mathfrak{H})^{\prime \prime}$ by the BorchersArveson theorem $[4,5,7]$.

Furthermore $\omega$ is a $\tau$-ground state if, and only if, for each pair $A, B \varepsilon \mathfrak{U}$ there exists a function $F_{A, B}$ which is continuous in $\operatorname{Im} z \geqq 0$, analytic and bounded in $\operatorname{Im} z>0$ and such that

$$
F_{A, B}(t)=\omega\left(A \tau_{t}(B)\right) \text {. }
$$

Again we will write $F_{A, B}(z)=\omega\left(A \tau_{z}(B)\right)$ even if $B \notin \mathfrak{U}_{\tau}$.

Similar characterizations of ceiling states are possible but in the first one has $H_{\omega} \leqq 0$, and in the second $\operatorname{Im} z \leqq 0$. We will concentrate on ground states and omit further reference to the entirely analogous ceiling states. 
There are several distinct notions of stability for an extremal $(\tau, \beta)$-KMS state. The strongest notion is the existence of a $\left(\tau^{P}, \beta\right)$-KMS vector state. But it was shown in [16] that the ground state of the ideal Fermi gas does not have a $\tau^{P}$ ground state as a normal state for a certain class of perturbations of the form $P=a^{*}(f) a(f)$. [We use the standard notation $a(f)$ etc. for the annihilation operators etc. which generate the CAR algebra describing the Fermi gas.] The failure of this form of stability has a clear physical origin. The perturbation causes the formation of an infinite number of infra-particles, i.e. particles with infinitesimally small energy, whenever $f$ fails to vanish on the Fermi surface $p^{2}=\mu$ and the ground state of the perturbed system has infinite density.

A second notion of stability is given by the condition

(*) $\quad \lim _{T \rightarrow \infty} \int_{-T}^{T} d t \omega\left(\left[A, \tau_{t}(P)\right]\right)=0$

for all $A, P \in \mathfrak{A}$, derived in Theorem 2 for $(\tau, \beta)$-KMS states. If $\omega$ is a strongly clustering ground state, and the Arveson spectrum, [4], of $P$ does not contain zero, then the condition $(*)$ still holds, but it does not hold for general $A, P$ in this case unless the Hamiltonian has an energy gap at zero energy. This is a consequence of the following theorem. In particular, condition $(*)$ fails to hold generally for the free Fermi sea, as one can also verify directly for $A=a(f), P=a^{*}(f)$, where $f$ is an $L^{2}$-function such that $\hat{f}(r)$ is sufficiently ugly near the Fermi surface $p^{2}=\mu$. Thus the infra-red divergence is the sole root of instability of this second type for strongly clustering ground states.

Theorem 3. Let $(\mathfrak{H}, \tau)$ be a $C^{*}$-, or $W^{*}$-, dynamical system and $\omega$ a strongly clustering $\tau$-ground state. Let $U_{\omega}(t)=e^{i t H_{\omega}}$ be the unitary group which implements $\tau$ in the cyclic representation $\left\{\mathscr{H}_{\omega}, \Pi_{\omega}, \Omega_{\omega}\right\}$.

The following conditions are equivalent

(*) 1. $\lim _{T \rightarrow \infty} \int_{-T}^{T} d t \omega\left(\left[A, \tau_{t}(B)\right]\right)=0$

for all $A, B \in \mathfrak{A}$.

2. $\lim _{T \rightarrow \infty} \int_{-T}^{T} d t\left\{\omega\left(A \tau_{t}(B)\right)-\omega(A) \omega(B)\right\}=0$

for all $A, B \in \mathfrak{A}$.

3. $\lim _{T \rightarrow \infty} \int_{-T}^{T} d t\left\{\left(\psi, U_{\omega}(t) \psi\right)-\left(\psi, \Omega_{\omega}\right)\left(\Omega_{\omega}, \psi\right)\right\}=0$

for all $\psi \in \mathscr{H}_{\omega}$.

4. There exists an $\varepsilon>0$ such that

$$
\sigma\left(H_{\omega}\right) \subseteq\{0\} \cup[\varepsilon, \infty\rangle,
$$

where $\sigma\left(H_{\omega}\right)$ denotes the spectrum of $H_{\omega}$.

Proof. $3 \Rightarrow 2 \Rightarrow 1$ trivially. We next prove $1 \Rightarrow 3$. It evidently suffices to consider $\psi$ such that $\left(\psi, \Omega_{\omega}\right)=0$. Now, as $\omega$ is strongly clustering it is pure and $\left(\mathscr{H}_{\omega}, \Pi_{\omega}\right)$ is 
irreducible. Therefore one may use the Kadison transitivity theorem (see, for example, [9, p. 44]) to construct an $A \in \mathfrak{U}$ such that $\Pi_{\omega}(A) \Omega_{\omega}=\psi$ and $\Pi_{\omega}\left(A^{*}\right) \Omega_{\omega}=0$. Therefore

$$
\begin{aligned}
\lim _{T \rightarrow \infty} \int_{-T}^{T} d t\left(\psi, U_{\omega}(t) \psi\right) & =\lim _{T \rightarrow \infty} \int_{-T}^{T} d t \omega\left(\left[A^{*}, \tau_{t}(A)\right]\right) \\
& =0 .
\end{aligned}
$$

$3 \Rightarrow 4$. Let $H$ be the restriction of $H_{\omega}$ to a closed separable $U_{\omega}$-invariant subspace $\mathscr{H}$ of $\mathscr{H}_{\omega} \ominus \mathbb{C} \Omega_{\omega}, H=\int_{0}^{\infty} r d E(r)$ the spectral decomposition of $H$, and $d v$ the measure in the corresponding spectral representation. Then for $\psi, \varphi \in \mathscr{H}$ we have

$$
\begin{aligned}
\int_{-T}^{T} d t\left(\psi, e^{i t H} \varphi\right) & =\int d t \chi_{T}(t)\left(\psi, e^{i t H} \varphi\right) \\
& =\int \frac{2 \sin (T p)}{p} d(\psi, E(p) \varphi) .
\end{aligned}
$$

Hence, assumption 3 implies that

$$
\lim _{T \rightarrow \infty} \int_{0}^{\infty} \frac{2 \sin (T p)}{r} g(r) d v(r)=0
$$

for all $g \in L^{1}(d v)$. But the uniform boundedness theorem then implies that the functions

$$
r \mapsto \frac{2 \sin \left(T_{n} r\right)}{r}
$$

are uniformly bounded in $L^{\infty}(d v)$ for all sequences $T_{n}$ such that $T_{n} \rightarrow \infty$. It follows immediately that $v([0, \varepsilon\rangle)=0$ for some $\varepsilon>0$, or

$$
\sigma(H) \cong[\varepsilon, \infty\rangle .
$$

A priori, $\varepsilon$ depends on the choice of the separable $U_{\omega}$-invariant subspace $\mathscr{H}$ of $\mathscr{H}_{\omega} \ominus \mathbb{C} \Omega_{\omega}$, but as the closed subspace generated by a countable number of such subspaces is still a separable $U_{\omega}$-invariant subspace, it follows by an ad absurdum argument that one may choose an $\varepsilon>0$ independent of the subspace.

$4 \Rightarrow 3$. By restricting to a separable, $U_{\omega}$-invariant subspace we may assume that $\mathscr{H}_{\omega}$ is separable. Keeping the notation above, the strong clustering assumption means that

$$
\lim _{t \rightarrow \pm \infty} \int_{\varepsilon}^{\infty} e^{i t r} f(r) d v(r)=0
$$

for all $f \in L^{1}(d v)$.

But if $g \in L^{1}(d v)$ then $f(r)=\frac{g(r)}{r}$ is an $L^{1}$-function since $\varepsilon>0$, and hence

$$
\lim _{T \rightarrow \infty} \int_{\varepsilon}^{\infty} \frac{2 \sin (T r)}{r} g(r) d v(r)=0
$$


that is

$$
\lim _{T \rightarrow \infty} \int_{-T}^{T} d t\left(\psi, e^{i t H} \varphi\right)=0
$$

for all $\psi, \varphi \in \mathscr{H}_{\omega} \ominus \mathbb{C} \Omega_{\omega}$.

Note that the implications $1 \Leftrightarrow 2 \Leftrightarrow 3 \Leftrightarrow 4$ in Theorem 3 remains valid when the strong clustering assumption for $\omega$ is replaced by the assumption of extremality among the $\tau$-ground states on $\tilde{\mathfrak{U}}=\mathbb{C} \mathbb{1}+\mathfrak{A}$. We preferred the strong clustering hypothesis because this is sufficient to derive condition 1 for $(\tau, \beta)$-KMS states with $\beta \in \mathbb{R}$.

Although the stability condition $(*)$ is not valid in general for ground states it is possible that it holds for all $A, P$ in a norm dense *-subalgebra $\mathfrak{U}_{0}$ of $\mathfrak{A}$. We have not been able to establish this except for $C^{*}$-dynamical systems which satisfy a strong condition of asymptotic abelianness.

A $C^{*}$-system $(\mathfrak{U}, \tau)$ is defined to be $L^{1}\left(\mathfrak{U}_{0}\right)$-asymptotically abelian if

$$
\int_{-\infty}^{\infty} d t\left\|\left[A, \tau_{t}(B)\right]\right\|<+\infty
$$

for all $A, B \in \mathfrak{A}_{0}$ where $\mathfrak{U}_{0}$ is a norm dense *-subalgebra of $\mathfrak{A}$. This type of condition first appeared in [16] where it was used to establish the existence of the Møller morphisms

$$
\gamma_{ \pm}(A)=\lim _{t \rightarrow \pm \infty} \tau_{-t}^{P} \tau_{t}(A)
$$

for $P \in \mathfrak{A}_{0}$. It was verified in [16] for the CAR algebra over $L^{2}\left(\mathbb{R}^{v}\right)$ and the free evolution.

Theorem 4. Let $(\mathfrak{H}, \tau)$ be an $L^{1}\left(\mathfrak{U}_{0}\right)$-asymptotically abelian $C^{*}$-dynamical system. It follows that

$$
\int_{-\infty}^{\infty} d t \omega\left(\left[A, \tau_{t}(B)\right]\right)=0
$$

for all $A, B \in \mathfrak{A}_{0}$, and for all $(\tau, \beta)$-KMS states where $\beta \in \mathbb{R} \cup\{ \pm \infty\}$.

Proof. First adjoin an identity, if necessary. One can extend $\tau$ to $\tilde{\mathfrak{U}}=\mathbb{C} \mathbb{1}+\mathfrak{A}$ by

$$
\tilde{\tau}_{t}((\alpha, A))=\left(\alpha, \tau_{t}(A)\right) ; \quad(\alpha, A) \in \tilde{\mathfrak{U}}
$$

and a straightforward argument with an approximate identity gives a one-to-one correspondence between the $(\tau, \beta)$-KMS states of $(\mathfrak{U}, \tau)$ and the $(\tilde{\tau}, \beta)$-KMS states $\tilde{\omega}$ of $(\tilde{\mathfrak{A}}, \tilde{\tau})$ with $\|\tilde{\omega} \mid \mathfrak{I}\|=1$. Thus we can assume that $\mathfrak{U}$ has an identity.

If $\beta=0$ then $\omega$ is a trace and $(*)$ is trivially true.

Next assume $\beta \in \mathbb{R} \backslash\{0\}$ and $\omega$ is an extremal $(\tau, \beta)$-KMS state. Therefore $\omega$ is a factor state and as $(\mathfrak{H}, \tau)$ is asymptotically abelian in norm it follows that it is strongly clustering, [7]. Thus the stability condition $(*)$ follows from Theorem 2 and it extends directly to finite convex combinations of extremal $(\tau, \beta)$-KMS states. Finally because the $L^{1}\left(\mathfrak{H}_{0}\right)$-asymptotic abelianness provides a uniform bound one can extend (*), for $A, B \in \mathfrak{U}_{0}$, to all weak* limit points of finite convex combinations 
of extremal states. But the Krein-Milman theorem allows any $(\tau, \beta)$-KMS state to be approximated in this manner and this completes the proof for finite $\beta$.

The cases $\beta= \pm \infty$ are identical so we assume $\beta=+\infty$.

First for $\varepsilon>0$ define $\chi_{\varepsilon}$ by specifying its Fourier transform $\hat{\chi}_{\varepsilon}$ through $\hat{\chi}_{\varepsilon}(p)$ $=(2 \pi)^{-1 / 2} / \varepsilon$ for $p \in[\varepsilon, 2 \varepsilon]$ and $\hat{\chi}_{\varepsilon}(p)=0$ otherwise, i.e. by Fourier transformation

$$
\chi_{\varepsilon}(t)=\int_{\varepsilon}^{2 \varepsilon} d p e^{i p t} / \varepsilon=e^{3 i \varepsilon t / 2}\left(\sin \frac{\varepsilon t}{2}\right) /\left(\frac{\varepsilon t}{2}\right) .
$$

Hence

$$
\lim _{\varepsilon \rightarrow 0} \chi_{\varepsilon}(t)=1 .
$$

But it then follows from the Lebesgue dominated convergence theorem, $L^{1}\left(\mathfrak{A}_{0}\right)$ asymptotic abelianness, and Fourier transformation that

$$
\begin{aligned}
\int_{-\infty}^{\infty} d t \omega\left(\left[A, \tau_{t}\left(A^{*}\right)\right]\right) & =\lim _{\varepsilon \rightarrow 0} \int_{-\infty}^{\infty} d t \chi_{\varepsilon}(t) \omega\left(\left[A ; \tau_{t}\left(A^{*}\right)\right]\right) \\
& =\lim _{\varepsilon \rightarrow 0} \int_{\varepsilon}^{2 \varepsilon} d\left(\Pi_{\omega}\left(A^{*}\right) \Omega_{\omega}, E_{\omega}(p) \Pi_{\omega}\left(A^{*}\right) \Omega_{\omega}\right) \\
& \geqq 0
\end{aligned}
$$

for all $A \in \mathfrak{A}_{0}$. We have used $E_{\omega}$ to denote the spectral measure associated with the unitary group $U_{\omega}$ which implements $\tau$ and the second step follows because the support of $E_{\omega}$ is in $[0, \infty\rangle$. But an identical argument with $\hat{\chi}_{\varepsilon}$ supported by $[-2 \varepsilon, \varepsilon]$ gives

$$
\begin{aligned}
\int_{-\infty}^{\infty} d t \omega\left(\left[A, \tau_{t}\left(A^{*}\right)\right]\right) & =-\lim _{\varepsilon \rightarrow 0} \int_{-2 \varepsilon}^{-\varepsilon} d\left(\Pi_{\omega}(A) \Omega_{\omega}, E_{\omega}(p) \Pi_{\omega}(A) \Omega_{\omega}\right) \\
& \leqq 0 .
\end{aligned}
$$

Therefore

$$
\int_{-\infty}^{\infty} d t \omega\left(\left[A, \tau_{t}\left(A^{*}\right)\right]\right)=0
$$

for all $A \in \mathfrak{A}_{0}$ and the general result follows by polarization.

We end this section with a discussion of the stability condition proposed in [10]. If $(\mathfrak{H}, \tau)$ is an asymptotic abelian $C^{*}$-dynamical system and $\omega$ is a $\tau$ stationary state of $\mathfrak{A}$, this stability condition is that for $P=P^{*} \in \mathfrak{A}_{0}$ and $\lambda \in \mathbb{R}$ sufficiently small (depending on $P$ ) there exists a $\tau^{\lambda P}$-stationary state $\omega^{\lambda P}$ on $\mathfrak{A}$ such that

$$
\lim _{\lambda \rightarrow 0} \omega^{\lambda P}(A)=\omega(A)
$$

for all $A \in \mathfrak{A}$, and the stability property

$$
\lim _{t \rightarrow \pm \infty} \omega^{\lambda P}\left(\tau_{t}(A)\right)=\omega(A)
$$

holds for all $A \in \mathfrak{Q}$. If $\omega$ is a strongly clustering $(\tau, \beta)$-KMS state these conditions are satisfied with $\omega^{\lambda P}$ equal to the normal state of Theorem 1 . Next assume that $\omega$ 
is a strongly clustering ground state satisfying the stability requirements of Theorem 3, i.e.

$$
\sigma\left(H_{\omega}\right) \cong\{0\} \cup[\varepsilon, \infty\rangle
$$

for some $\varepsilon>0$. It follows easily from resolvent identities that $H_{\omega}+\lambda \Pi_{\omega}(P)$ has a unique normalized eigenvector $\psi^{\lambda P}$ (up to a phase) of minimum energy for small enough $\lambda$, and one may verify that the corresponding vector-states $\omega^{\lambda P}$ satisfies the stability properties above.

In the case that $\omega$ is a strongly clustering ground state, but $H_{\omega}$ has no energy gap at zero, the existence of normal perturbed ground states $\omega^{\lambda P}$ does not necessarily follow. We have already remarked that the free Fermi sea is an example of this, [16]. However, the ground state of the free Fermi gas is unique [7]. It follows from the next Proposition that this ground state is stable in the sense of [10].

Proposition 5. Let $(\mathfrak{H}, \tau)$ be a $C^{*}$-dynamical system where $\mathfrak{A}$ has an identity and which is $L^{1}\left(\mathfrak{U}_{0}\right)$-asymptotic abelian. Assume that $(\mathfrak{H}, \tau)$ has a unique ground state $\omega$.

It follows that for each $P=P^{*} \in \mathfrak{A}_{0}$ there exists a ground state $\omega^{P}$ for $\left(\mathfrak{H}, \tau^{P}\right)$. If $\left\{\omega^{P}\right\}$ is an arbitrary family of such ground states, then

$$
\lim _{\lambda \rightarrow 0} \omega^{\lambda P}(A)=\omega(A)
$$

and

$$
\lim _{t \rightarrow \pm \infty} \omega^{P}\left(\tau_{t}(A)\right)=\omega(A)
$$

for all $A \in \mathfrak{A}, P=P^{*} \in \mathfrak{A}_{0}$.

Proof. Since $H_{\omega}+\Pi_{\omega}(P)$ is bounded below for all $P=P^{*} \in \mathfrak{A}$, the existence of a ground state for $\tau^{P}$ follows by taking a weak* limit of a sequence of vector states, where the $H_{\omega}+\Pi_{\omega}(P)$-spectrum of the vectors tend to the infinum of the spectrum of $H_{\omega}+\Pi_{\omega}(P),[7]$. Let $\omega^{P}$ be an arbitrary ground state of $\left(\mathfrak{Q}, \tau^{P}\right)$ for each $P$.

The assumed asymptotic abelianness ensures that the Møller scattering morphisms

$$
\gamma_{ \pm}^{P}(A)=\lim _{t \rightarrow \pm \infty} \tau_{-t}^{P} \tau_{t}(A)
$$

exist for $P=P^{*} \in \mathfrak{A}_{0},[16] . \gamma_{ \pm}^{P}$ define ${ }^{*}$-isomorphisms between $\mathfrak{A}$ and $\gamma_{ \pm}^{P}(\mathfrak{U})$ satisfying the intertwining relations

$$
\gamma_{ \pm}^{P} \tau_{t}=\tau_{t}^{P} \gamma_{ \pm}^{P}
$$

for all $t \in \mathbb{R}$. Hence the $C^{*}$-dynamical systems $\left(\gamma_{ \pm}^{P}(\mathfrak{H}), \tau^{P}\right)$ have a unique ground state $\varrho_{ \pm}^{P}$, which are defined by $\varrho_{ \pm}^{P}{ }^{\circ} \gamma_{ \pm}^{P}=\omega$. Since $\omega^{P} \mid \gamma_{ \pm}^{P}(\mathfrak{I})$ is a ground state, it follows that

$$
\omega^{P} \circ \gamma_{ \pm}^{P}=\omega
$$

and hence

$$
\omega(A)=\omega^{P} \circ \gamma_{ \pm}^{P}(A)=\lim _{t \rightarrow \pm \infty} \omega^{P}\left(\tau_{t}(A)\right) .
$$


Now, by the estimate

$$
\left\|\gamma_{+}^{\lambda P}(A)-A\right\| \leqq|\lambda| \int_{0}^{\infty} d s\left\|\left[P, \tau_{t}(A)\right]\right\|
$$

which is valid for $A \in \mathfrak{A}_{0}$, it follows that

$$
\lim _{\lambda \rightarrow 0} \gamma_{+}^{\lambda P}(A)=A
$$

for all $A \in \mathfrak{A}$. Combining this with $\omega^{\lambda P}\left(\gamma_{+}^{\lambda P}(A)\right)=(A)$, it follows that

$$
\lim _{\lambda \rightarrow 0} \omega^{\lambda P}(A)=\omega(A) \text {. }
$$

Later on, in Corollary 7, we will discuss a fourth stability condition which is equivalent with the one considered in Theorem 4.

\section{Stability and the KMS Condition}

In the previous section we studied various stability properties of KMS states and our next aim is to derive a result in the converse direction, i.e. we establish a result of the Haag, Kastler, Trych-Pohlmeyer type. Roughly we deduce that the extremal $\tau$-invariant states of asymptotically abelian systems which are stable under local perturbations are necessarily extremal KMS states at some value of $\beta \in \mathbb{R} \cup\{ \pm \infty\}$. In fact we assume $L^{1}$-asymptotic abelianness and a three point cluster property. Within the framework of $L^{1}$-asymptotic abelianness the following theorem essentially characterizes extremal $(\tau, \beta)$-KMS states with a slight discrepancy if $\beta=0$. This discrepancy arises because the extremal $(\tau, 0)$-KMS states, i.e. the extremal invariant traces, are not necessarily factor states, but this discrepancy is absent if $\mathfrak{A}$ has a unique trace state. (This theorem was claimed to be true in [11], but the proof was not completed. For elementary results of extremal invariant states over asymptotically abelian systems etc., see [7].)

Theorem 6. Let $(\mathfrak{H}, \tau)$ be a $C^{*}$-dynamical system which is $L^{1}\left(\mathfrak{A}_{0}\right)$-asymptotically abelian, and $\omega$ a $\tau$-invariant state over $\mathfrak{H}$. Assume that

1. Either

$$
\begin{aligned}
& \lim _{\substack{i n f \\
i \neq j}} \omega\left(t_{i}-t_{j} \mid \rightarrow \infty\right. \\
& =\omega\left(\tau_{t_{1}}\left(A_{1}\right) \tau_{t_{2}}\left(A_{2}\right) \tau_{t_{3}}\left(A_{3}\right)\right) \\
& =\omega\left(A_{2}\right) \omega\left(A_{3}\right)
\end{aligned}
$$

for all $A_{1}, A_{2}, A_{3} \in \mathfrak{A}$, or $\omega$ is a factor state.

2. $\omega$ satisfies the stability requirement

$$
\int_{-\infty}^{\infty} d t \omega\left(\left[P, \tau_{t}(A)\right]\right)=0
$$

for all $A, P \in \mathfrak{U}_{0}$.

It follows that $\omega$ is an extremal $(\tau, \beta)$-KMS state for some $\beta \in \mathbb{R} \cup\{ \pm \infty\}$.

Proof. As $t \mapsto \tau_{t}(B)$ is uniformly continuous for each $B \in \mathfrak{A}_{0}$, it follows from the $L^{1}\left(\mathfrak{U}_{0}\right)$ asymptotic abelianness that

$$
\lim _{t \rightarrow \pm \infty}\left\|\left[A, \tau_{t}(B)\right]\right\|=0
$$


for all $A, B \in \mathfrak{U}_{0}$, and hence for all $A, B \in \mathfrak{A}$. Thus if $\omega$ is a factor state it follows that

$$
\begin{aligned}
& \lim _{\substack{i \neq j \\
i \neq j}} \omega\left(\tau_{t_{i}-t_{j} \mid \rightarrow \infty}\left(\mathrm{A}_{1}\right) \ldots \tau_{t_{n}}\left(A_{n}\right)\right) \\
& \quad=\omega\left(A_{1}\right) \ldots \omega\left(A_{n}\right)
\end{aligned}
$$

for all $n \in \mathbb{Z}_{+}$and all $A_{i} \in \mathfrak{U}$. Therefore it suffices to prove the theorem under the three point clustering assumption.

If $\left\{E_{\alpha}\right\}$ is an approximate unit on $\mathfrak{U}$ it follows that

$$
\lim _{\alpha} \Pi_{\omega}^{\omega}\left(E_{\alpha}\right) \Omega_{\omega}=\Omega_{\omega}
$$

and using this one easily proves the two point cluster property

$$
\lim _{t \rightarrow \pm \infty} \omega\left(A \tau_{t}(B)\right)=\omega(A) \omega(B)
$$

from the corresponding three point property. Also, we may assume that $\mathfrak{A}_{0}$ is closed under regularization by $L^{1}$-functions.

Let us define

$$
\begin{aligned}
& F_{i}(t)=F_{A_{i}, P_{i}}(t)=\omega\left(P_{i} \tau_{t}\left(A_{i}\right)\right)-\omega\left(P_{i}\right) \omega\left(A_{i}\right) \\
& G_{i}(t)=G_{A_{i}, P_{i}}(t)=\omega\left(\tau_{t}\left(A_{i}\right) P_{i}\right)-\omega\left(A_{i}\right) \omega\left(P_{i}\right)
\end{aligned}
$$

for $A_{i}, P_{i} \in \mathfrak{A}_{0}$.

Observation 1 (Haag and Trych-Pohlmeyer [11]). $t \mapsto F_{1}(t) F_{2}(t)-G_{1}(t) G_{2}(t)$ is an $L^{1}$-function, and

$$
\int_{-\infty}^{\infty} d t\left\{F_{1}(t) F_{2}(t)-G_{1}(t) G_{2}(t)\right\}=0
$$

Proof. By the stability requirement we have

$$
\begin{aligned}
0 & =\int_{-\infty}^{\infty} d t \omega\left(\left[P_{1} \tau_{s}\left(P_{2}\right), \tau_{t}\left(A_{1} \tau_{s}\left(A_{2}\right)\right)\right]\right) \\
& =I_{1}(s)+I_{2}(s)+I_{3}(s)+I_{4}(s)
\end{aligned}
$$

where

$$
\begin{aligned}
& I_{1}(s)=\int_{-\infty}^{\infty} d t \omega\left(P_{1}\left[\tau_{s}\left(P_{2}\right), \tau_{t}\left(A_{1}\right)\right] \tau_{t+s}\left(A_{2}\right)\right) \\
& I_{2}(s)=\int_{-\infty}^{\infty} d t \omega\left(P_{1} \tau_{t}\left(A_{1}\right)\left[\tau_{s}\left(P_{2}\right), \tau_{s+t}\left(A_{2}\right)\right]\right) \\
& I_{3}(s)=\int_{-\infty}^{\infty} d t \omega\left(\left[P_{1}, \tau_{t}\left(A_{1}\right)\right] \tau_{t+s}\left(A_{2}\right) \tau_{s}\left(P_{2}\right)\right) \\
& I_{4}(s)=\int_{-\infty}^{\infty} d t \omega\left(\tau_{t}\left(A_{1}\right)\left[P_{1}, \tau_{t+s}\left(A_{2}\right)\right] \tau_{s}\left(P_{2}\right)\right) .
\end{aligned}
$$

The integrands of $I_{2}$ and $I_{3}$ are dominated by $L^{1}$-functions which are independent of $s$, and using the two-point clustering and Lebesgues dominated convergence 
theorem, we obtain

$$
\begin{aligned}
\lim _{s \rightarrow \infty}\left\{I_{2}(s)+I_{3}(s)\right\} \\
=\int_{-\infty}^{\infty} d t \omega\left(P_{1} \tau_{t}\left(A_{1}\right)\right) \omega\left(\left[P_{2}, \tau_{t}\left(A_{2}\right)\right]\right) \\
\quad+\int_{-\infty}^{\infty} d t \omega\left(\left[P_{1}, \tau_{t}\left(A_{1}\right)\right]\right) \omega\left(\tau_{t}\left(A_{2}\right) P_{2}\right) \\
=\int_{-\infty}^{\infty} d t\left\{F_{A_{1}, P_{1}}(t) F_{A_{2}, P_{2}}(t)-G_{A_{1}, P_{1}}(t) G_{A_{2}, P_{2}}(t)\right\},
\end{aligned}
$$

where the last step used the stability condition.

Hence it is enough for Observation 1 to show that

$$
\lim _{s \rightarrow \infty} I_{1}(s)=\lim _{s \rightarrow \infty} I_{4}(s)=0 .
$$

But by a change of variable,

$$
I_{1}(s)=\int_{-\infty}^{\infty} d t \omega\left(\tau_{-s}\left(P_{1}\right)\left[P_{2}, \tau_{t}\left(A_{1}\right)\right] \tau_{t+s}\left(A_{2}\right)\right) .
$$

Again the integrand is dominated by the $L^{1}$-function $t \mapsto\left\|P_{1}\right\| \cdot\left\|\left[P_{2}, \tau_{t}\left(A_{1}\right)\right]\right\|\left\|A_{2}\right\|$ which is independent of $s$, and the limit of the integrand as $s \rightarrow \infty$ is

$$
\omega\left(P_{1}\right) \omega\left(\left[P_{2}, \tau_{t}\left(A_{1}\right)\right]\right) \omega\left(A_{2}\right)
$$

by the three point cluster property. Thus, by Lebesgue theorem,

$$
\begin{aligned}
\lim _{s \rightarrow \infty} I_{1}(s) & =\omega\left(P_{1}\right) \omega\left(A_{2}\right) \int_{-\infty}^{\infty} d t \omega\left(\left[P_{2}, \tau_{t}\left(A_{1}\right)\right]\right) \\
& =0 .
\end{aligned}
$$

One shows that $\lim _{s \rightarrow \infty} I_{4}(\mathrm{~s})=0$ by a similar reasoning, and this ends the proof of Observation 1.

Now, from the relations

$$
\begin{aligned}
F_{A, P}(t) & =G_{P, A}(-t) \\
F_{\tau_{s}(A), P}(t) & =F_{A, P}(t+s)
\end{aligned}
$$

and Observation 1 it follows that

$$
\int d t\left\{F_{1}(t) G_{2}(s-t)-G_{1}(t) F_{2}(s-t)\right\}=0 .
$$

Define $H_{1}=H_{A_{1}, P_{1}}$ by

$$
H_{1}(t)=F_{1}(t)-G_{1}(t) .
$$

Then $H_{1}$ is an $L^{1}$-function, and from the relation above we get

$$
\begin{aligned}
& \int d t\left\{H_{1}(t) G_{2}(s-t)-G_{1}(t) H_{2}(s-t)\right\}=0 \\
& \int d t\left\{F_{1}(t) H_{2}(s-t)-H_{1}(t) F_{2}(s-t)\right\}=0 .
\end{aligned}
$$


If $U_{\omega}(t)=\int e^{i p t} d E_{\omega}(p)$ is the spectral decomposition of $U_{\omega}$, let

$$
\mu_{i}=\mu_{A i, P_{i}}, \quad v_{i}=v_{A_{i}, P_{i}}
$$

be the measures defined by

$$
\begin{aligned}
& d \mu_{i}(p)=\left(P_{i}^{*} \Omega_{\omega}, d E_{\omega}(p) A_{i} \Omega_{\omega}\right)-\omega\left(P_{i}\right) \omega\left(A_{i}\right) \delta(p) d p \\
& d v_{i}(p)=\left(A_{i}^{*} \Omega_{\omega}, d E_{\omega}(-p) P_{i} \Omega_{\omega}\right)-\omega\left(A_{i}\right) \omega\left(P_{i}\right) \delta(p) d p .
\end{aligned}
$$

Then

$$
\begin{aligned}
& F_{i}(t)=\int e^{i t p} d \mu_{i}(p) \\
& G_{i}(t)=\int e^{i t p} d v_{i}(p)
\end{aligned}
$$

and thus

$$
d \mu_{i}(p)-d v_{i}(p)=\hat{H}_{i}(p) d p,
$$

where $\hat{H}_{i}$ is the Fourier transform of $H_{i}$. From the convolution relations above we now obtain

Observation 2.

$$
\begin{aligned}
& \hat{H}_{2}(p) d v_{1}(p)=\hat{H}_{1}(p) d v_{2}(p) \\
& \hat{H}_{2}(p) d \mu_{1}(p)=\hat{H}_{1}(p) d \mu_{2}(p)
\end{aligned}
$$

for all $A_{i}, P_{i} \in \mathfrak{A}_{0}$.

Next, define a subset $S \cong \mathbb{R}$ by

$S=\left\{p \in \mathbb{R} ; \hat{H}_{\dot{A}, P}(p) \neq 0\right.$ for some $\left.A, P \in \mathfrak{U}_{0}\right\}$.

Observation 3. $S$ is an open set, $S=-S$ and $0 \notin S$.

Proof. $S$ is open since each $\hat{H}_{A, P}$ is the Fourier transform of an $L^{1}$-function, and thus is continuous. The symmetry follows from the relation

$$
\begin{aligned}
d p \hat{H}_{A, P}(p)= & \left(P^{*} \Omega_{\omega}, d E_{\omega}(p) A \Omega_{\omega}\right) \\
& -\left(A^{*} \Omega_{\omega}, d E_{\omega}(-p) P \Omega_{\omega}\right) \\
= & -d p \hat{H}_{P, A}(-p)
\end{aligned}
$$

and $\hat{H}_{A, P}(0)=0$ by this same relation (or stability).

Observation 4. There exists a well defined pair $\mu, v$ of $\sigma$-finite measures on $S$ such that

$$
\begin{aligned}
& d \mu_{A, P}(p)=\hat{H}_{A, P}(p) d \mu(p) \\
& d v_{A, P}(p)=\hat{H}_{A, P}(p) d v(p)
\end{aligned}
$$


for $p \in S$, and, furthermore

$$
d \mu(p)=-d v(-p) .
$$

Proof. Since $S$ is open, $S$ is the union of an increasing sequence of compact sets, and as each $\hat{H}_{A, P}$ is continuous it follows that there exists a countable partition $\left\{S_{1}, S_{2}, \ldots\right\}$ of $S$ into Borel sets, and elements $A_{i}, P_{i} \in \mathfrak{A}_{0}$ such that

$$
\left|\hat{H}_{A_{\imath}, P_{\imath}}(p)\right| \geqq 1
$$

for $p \in S_{i}$. Now, define

$$
\begin{array}{r}
d \mu(p)=\frac{d \mu_{A_{i}, P_{i}}(p)}{\hat{H}_{A_{\imath}, P_{\imath}}(p)} \\
d \nu(p)=\frac{d v_{A_{i}, P_{\imath}}(p)}{\hat{H}_{A_{\imath}, P_{\imath}}(p)}
\end{array}
$$

for $p \in S_{i}, i=1,2, \ldots$. Then $\mu, v$ are well-defined $\sigma$-finite Borel measures on $S$, and Observation 2 implies that

$$
\begin{aligned}
d \mu_{A, P}(p) & =\hat{H}_{A, P}(p) d \mu(p) \\
d v_{A, P}(p) & =\hat{H}_{A, P}(p) d v(p)
\end{aligned}
$$

for all $A, P \in \mathfrak{A}_{0}$ and all $p \in S$. Finally

$$
d \mu(p)=\frac{d \mu_{A_{i}, P_{i}}(p)}{\hat{H}_{A_{\imath}, P_{l}}(p)}=\frac{d v_{P_{i}, A_{i}}(-p)}{-\hat{H}_{P_{\imath}, A_{\imath}}(-p)}=-d v(-p) .
$$

Now, the Radon-Nikodym theorem implies that there exists $|\mu|+|v|-$ measurable functions $\psi_{i}$ on $S$ such that

$$
\begin{aligned}
& d \mu(p)=\psi_{1}(p) d(|\mu|+|v|)(p) \\
& d v(p)=\psi_{2}(p) d(|\mu|+|v|)(p) .
\end{aligned}
$$

Since $d \mu(-p)=-d v(p)$ we have $d(|\mu|+|v|)(p)=d(|\mu|+|v|)(-p)$ and $\psi_{1}(-p)$ $=-\psi_{2}(p)$.

Define subsets $S_{0}, S_{\infty}$ and $S_{f}$ of $\mathbb{R}$ by

$$
\begin{aligned}
S_{0} & =\left\{p \in S ; \psi_{1}(p)=0\right\} \\
S_{\infty} & =\left\{p \in S ; \psi_{2}(p)=0\right\} \\
S_{f} & =\mathbb{R} \backslash\left(S_{0} \cup S_{\infty}\right) .
\end{aligned}
$$

Since $\psi_{1}(p)=0$ if, and only if, $\psi_{2}(-p)=0$, we deduce immediately

\section{Observation 5.}

$$
S_{0}=-S_{\infty}, \quad S_{f}=-S_{f}
$$

in the sense that the sets differ only on a set of $|\mu|+|v|$-measure zero. 
Define a Borel function $\varphi$ on $\mathbb{R} \backslash S_{\infty}$ by

$$
\varphi(p)=\left\{\begin{array}{ccc}
\frac{\psi_{1}(p)}{\psi_{2}(p)} & \text { on } & S \backslash S_{\infty} \\
1 & \text { on } & \mathbb{R} \backslash S .
\end{array}\right.
$$

Observation 6.

$$
d \mu_{A, P}(p)=\varphi(p) d v_{A, P}(p)
$$

on $\mathbb{R} \backslash S_{\infty}$. Furthermore $\varphi(p)>0$ and $\varphi(-p)=\varphi(p)^{-1}$ on $S_{f}$ (except for a set of $|\mu|+|v|$-measure zero).

Proof. It follows from Observation 4 that

$$
\begin{gathered}
d \mu_{A, P}(p)=\psi_{1}(p) \hat{H}_{A, P}(p) d(|\mu|+|v|)(p) \\
d v_{A, P}(p)=\psi_{2}(p) \hat{H}_{A, P}(p) d(|\mu|+|v|)(p)
\end{gathered}
$$

for $p \in S$, while

$$
d \mu_{A, P}(p)-d v_{A, P}(p)=\hat{H}_{A, P}(p) d p=0
$$

for $p \in S^{c}$. As $d \mu_{A^{*}, A}(p) \geqq 0$ and $d v_{A^{*}, A}(p) \geqq 0$ for all $p \neq 0$ and all $A \in \mathfrak{U}_{0}$, it follows that $\varphi(p) \geqq 0$ and since $\varphi(p) \neq 0$ for $p \in S_{f}$, one has $\varphi(p)>0$ for $p \in S_{f} . \varphi(p)=\varphi(-p)^{-1}$ is a consequence of $\psi_{1}(p)=-\psi_{2}(-p)$.

Observation 7. If $E_{\omega}$ is the projection valued measure corresponding to $H_{\omega}$, then

$$
E_{\omega}\left(S_{0}\right)=0
$$

and, consequently

$$
E_{\omega}\left(S_{\infty} \cup S_{f}\right)=\mathbb{1} .
$$

Proof. From the relation

$$
d \mu_{A, P}(p)=\psi_{1}(p) \hat{H}_{A, P}(p) d(|\mu|+|v|)(p)
$$

it follows that

$$
\mu_{A, P}\left(S_{0}\right)=0
$$

and hence, as $0 \notin S_{0}$ by Observation 3,

$$
\left(P^{*} \Omega_{\omega}, E_{\omega}\left(S_{0}\right) A \Omega_{\omega}\right)=\mu_{A, P}\left(S_{0}\right)=0
$$

for all $A, P \in \mathfrak{A}_{0}$.

Observation 8.

$$
\begin{aligned}
& \left(P^{*} \Omega_{\omega}, E_{\omega}\left(S_{f}\right) A \Omega_{\omega}\right) \\
& \quad=\left(\varphi\left(-H_{\omega}\right)^{1 / 2} A^{*} \Omega_{\omega}, \varphi\left(-H_{\omega}\right)^{1 / 2} P \Omega_{\omega}\right)
\end{aligned}
$$

for all $A, P \in \mathscr{M}_{\omega}=\Pi_{\omega}(\mathfrak{U})^{\prime \prime}$. 
Proof. By Observation 6, one has

$$
\begin{aligned}
\left(P^{*} \Omega_{\omega}, d E_{\omega}(p) A \Omega_{\omega}\right) \\
\quad=d \mu_{A, P}(p)+\omega(P) \omega(A) \delta(p) d p \\
\quad=\varphi(p) d v_{A, P}(p)+\omega(P) \omega(A) \delta(p) d p \\
\quad=\left(\varphi(p)^{1 / 2} A^{*} \Omega_{\omega}, d E_{\omega}(-p) \varphi(p)^{1 / 2} P \Omega_{\omega}\right)
\end{aligned}
$$

for $p \in \mathbb{R} \backslash S_{\infty}$, and $A, P \in \mathfrak{A}_{0}$, where we have identified $\mathfrak{A}$ and $\Pi_{\omega}(\mathfrak{H})$. Integrating over $\mathbb{R} \backslash S_{\infty}=S_{f} \cup S_{0}$, we obtain from spectral theory that

$$
\mathfrak{A}_{0} \Omega_{\omega} \subseteq D\left(\varphi\left(-H_{\omega}\right)^{1 / 2} E_{\omega}\left(-\left(S_{f} \cup S_{0}\right)\right)\right)
$$

and

$$
\begin{aligned}
\left(P^{*} \Omega_{\omega}, E_{\omega}\left(S_{f}\right) A \Omega_{\omega}\right) & =\left(P^{*} \Omega_{\omega}, E_{\omega}\left(S_{f} \cup S_{0}\right) A \Omega_{\omega}\right) \\
& =\left(\varphi\left(-H_{\omega}\right)^{1 / 2} A^{*} \Omega_{\omega}, \varphi\left(-H_{\omega}\right)^{1 / 2} P \Omega_{\omega}\right),
\end{aligned}
$$

where we have used $E_{\omega}\left(S_{0}\right)=0$ and $E_{\omega}\left(-\left(S_{f} \cup S_{0}\right)\right)=E_{\omega}\left(S_{f} \cup S_{\infty}\right)$ which follows from Observations 5 and 7 .

Since $\mathfrak{A}_{0}$ is strong *-dense in $\mathscr{M}_{\omega}$, this relation extends to all $A, P \in \mathscr{M}_{\omega}$.

Next define

$$
E_{f}=\left[\mathscr{M}_{\omega}^{\prime} \Omega_{\omega}\right] .
$$

Then $E_{f} \in \mathscr{M}_{\omega}$.

\section{Observation 9.}

$$
E_{\omega}\left(S_{f}\right)=E_{\omega}\left(S_{f}\right) E_{f}=E_{f} E_{\omega}\left(S_{f}\right) .
$$

Proof. Since

$$
e^{i t H_{\omega}} \mathscr{M}_{\omega}^{\prime} e^{-i t H_{\omega}}=\mathscr{M}_{\omega}^{\prime}
$$

for all $t \in \mathbb{R}$, it follows that. $E_{f}$ commutes with $e^{i t H_{\omega}}$, and hence

$$
E_{\omega}\left(S_{f}\right) E_{f}=E_{f} E_{\omega}\left(S_{f}\right) \text {. }
$$

By Observation 8 , one has

$$
\begin{aligned}
& \left(E_{\omega}\left(S_{f}\right)\left(\mathbb{1}-E_{f}\right) P^{*} \Omega_{\omega}, E_{\omega}\left(S_{f}\right)\left(\mathbb{1}-E_{f}\right) A \Omega_{\omega}\right) \\
& \quad=\left(\varphi\left(-H_{\omega}\right)^{1 / 2} A^{*}\left(\mathbb{1}-E_{f}\right) \Omega_{\omega}, \varphi\left(-H_{\omega}\right)^{1 / 2} P\left(\mathbb{1}-E_{f}\right) \Omega_{\omega}\right) \\
& \quad=0
\end{aligned}
$$

for all $A, P \in \mathscr{M}_{\omega}$, and hence

$$
E_{\omega}\left(S_{f}\right)\left(\mathbb{1}-E_{f}\right)=0 \text {. }
$$

Now, $\Omega_{\omega}$ is cyclic and separating for the von Neumann algebra $\mathscr{M}_{E}=E_{f} \mathscr{M}_{\omega} E_{f}$ on $\mathscr{H}_{E}=E_{f} \mathscr{H}_{\omega}$. Define $H_{E}=H_{\omega} E_{f}=E_{f} H_{\omega}$ and let $\Delta, J$ be the modular operator and modular conjugation associated with the pair $\left(\mathscr{M}_{E}, \Omega\right)$. Then

$$
H_{E}=\int p d E_{E}(p)=\int p d E_{\omega}(p) E_{f}
$$


is the spectral decomposition of $H_{E}$. As $e^{i t H_{E}}$ leaves $\Omega_{\omega}$ invariant and defines an automorphism group of $\mathscr{M}_{E}$, it follows that

$$
J H_{E} J=-H_{E}
$$

and hence

$$
J E_{E}(B) J=E_{E}(-B)
$$

for all Borel sets $B \subseteq \mathbb{R}$. In particular it follows from Observation 7 that

$$
\begin{aligned}
E_{f}=J E_{f} J & =J E_{E}\left(S_{f} \cup S_{\infty}\right) J \\
& =E_{E}\left(-\left(S_{f} \cup S_{\infty}\right)\right) .
\end{aligned}
$$

But

$$
E_{E}\left(-S_{\infty}\right)=E_{E}\left(S_{0}\right)=0
$$

by Observations 5 and 7. Thus

$$
E_{f}=E_{E}\left(-S_{f}\right)=E_{\omega}\left(S_{f}\right) E_{f}
$$

and Observation 9 implies :

Observation 10 .

$$
E_{f}=E_{\omega}\left(S_{f}\right)
$$

Now, by extremality and asymptotic abelianness $E_{\omega}=E_{\omega}(\{0\})$ is a onedimensional projection, and as $\Omega_{\omega}$ is separating for $\mathscr{M}_{E}$ it follows that

$$
\inf _{B^{\prime} \in \operatorname{Co} \tau_{\mathbb{R}^{(B)}}}\left|\omega\left(A B^{\prime} C\right)-\omega(B) \omega(A C)\right|=0
$$

for all $A, B, C \in \mathscr{M}_{E}$. As $H_{E}$ and $\triangle$ commute strongly, it follows from $[12,13]$ that the joint spectrum $\Sigma$ of $\left(\log \Delta, H_{E}\right)$ is a closed additive subset of $\mathbb{R}^{2}$. But since $\Omega_{\omega}$ is separating for $\mathscr{M}_{E}, \Sigma$ is symmetric, and we conclude:

Observation 11. The joint spectrum $\Sigma$ of $\left(\log \Delta, H_{E}\right)$ is a closed subgroup of $\mathbb{R}^{2}$.

We next show

Observation 12. If $E_{\omega}\left(S_{f}\right)$ is not one-dimensional, then $S_{f}$ is dense in $\mathbb{R}$ and $\sigma\left(H_{E}\right)=\mathbb{R}$.

Proof. $\sigma\left(H_{E}\right)$ is a group by the remarks before Observation 11, and as 0 is a simple eigenvalue of $H_{\omega}$ it follows from the assumption and $E_{\omega}\left(S_{f}\right)=E_{f}$ that $\sigma\left(H_{E}\right) \neq\{0\}$. But $\sigma\left(H_{E}\right)$ cannot have any nonzero isolated points because this would imply that $H_{\omega}$ has a nonzero eigenvalue $\lambda$ with a corresponding eigenvector $\psi$ such that

$$
U_{\omega}(t) \psi=e^{i \lambda t} \psi
$$


But then

$$
\begin{aligned}
\left(\psi, \Pi_{\omega}\left(\tau_{t}(B)\right) \Omega_{\omega}\right) & =\left(U_{\omega}(-t) \psi, \Pi_{\omega}(B) \Omega_{\omega}\right) \\
& =e^{i \lambda t}\left(\psi, \Pi_{\omega}(B) \Omega_{\omega}\right)
\end{aligned}
$$

for all $B \in \mathfrak{A}$. Therefore choosing $B$ such that

$$
\left(\psi, \Pi_{\omega}(B) \Omega_{\omega}\right) \neq 0
$$

this contradicts the fact that

$$
\lim _{t \rightarrow \infty} \Pi_{\omega}\left(\tau_{t}(B)\right)=\omega(B) \mathbb{1}
$$

in the weak topology. It follows that

$$
\sigma\left(H_{E}\right)=\mathbb{R}
$$

Observation 13.

$$
\sigma\left(H_{E}\right)+\sigma\left(H_{\mathbb{1}-E}\right) \cong \sigma\left(H_{\mathbb{1}-E}\right) .
$$

Proof. This is demonstrated as in the last part of the proof of Proposition 4.2 in [15].

Observation 14. The restrictions of the measures $d \mu_{A, P}$ and $d v_{A, P}$ to $S_{0} \cup S_{\infty}$ are absolutely continuous with respect to Lebesgue measure and

$$
\begin{aligned}
& d \mu_{A, P}(p)=\left\{\begin{array}{ll}
0 & ; \quad p \in S_{0} \\
\hat{H}_{A, P}(p) d p ; & p \in S_{\infty}
\end{array},\right. \\
& d v_{A, P}(p)=\left\{\begin{array}{cl}
-\hat{H}_{A, P}(p) d p ; & p \in S_{0} \\
0 \quad & ; \quad p \in S_{\infty} .
\end{array}\right.
\end{aligned}
$$

Proof. This follows from the relation

$$
d \mu_{A, P}(p)-d v_{A, P}(p)=\hat{H}_{A, P}(p) d p
$$

together with the equations

$$
\begin{array}{ll}
d \mu_{A, P}(p)=0 ; & p \in S_{0} \\
d v_{A, P}(p)=0 ; & p \in S_{\infty} .
\end{array}
$$

Next, define subsets $S_{ \pm} \subseteq \mathbb{R}$ by

$$
\begin{aligned}
& S_{+}=\bigcap_{A \in \mathfrak{Q}_{0}}\left\{p ; \hat{H}_{A, A^{*}}(p) \geqq 0\right\} \\
& S_{-}=\bigcap_{A \in \mathfrak{Q}_{0}}\left\{p ; \hat{H}_{A, A^{*}}(p) \leqq 0\right\} .
\end{aligned}
$$

Then $S_{ \pm}$are closed sets, and since

$$
S=\bigcup_{A \in \mathscr{A}_{0}}\left\{p ; \hat{H}_{A, A^{*}}(p) \neq 0\right\}
$$


by polarization, it follows that

$$
S_{+} \cap S_{-} \cap S=\emptyset .
$$

But the measures $d \mu_{A, A^{*}}(p)$ and $d v_{A, A^{*}}(p)$ are non-negative for $p \neq 0$, and hence it follows from Observation 14 that

$$
S_{\infty} \subseteq S_{+}, \quad S_{0} \subseteq S_{-}
$$

except for sets of spectral measure zero. By subtracting the latter sets from $S_{\infty}$ and $S_{0}$ we may assume that the inclusions are strict and hence

$$
\overline{S_{\infty}} \cong S_{+}, \quad \overline{S_{0}} \cong S_{-},
$$

where the bar denotes closure. Therefore one has:

Observation 15.

$$
\overline{S_{\infty}} \cap \overline{S_{0}} \cap S \cong S_{+} \cap S_{-} \cap S=\emptyset .
$$

Now, by Observation 7 and 10, one has

$$
E_{\omega}\left(S_{\infty}\right)=\mathbb{1}-E_{f}
$$

and thus

Observation 16.

$$
\sigma\left(H_{1-E}\right) \cong \overline{S_{\infty}} .
$$

We now finish the proof of Theorem 6 . We consider two cases

Case 1. $E_{\omega}\left(S_{f}\right)$ is not one-dimensional. In this case $\sigma\left(H_{E}\right)=\mathbb{R}$ by Observation 12 . We show, ad absurdum, that $E_{f}=\mathbb{1}$. If not $\sigma\left(H_{1-E}\right)=\mathbb{R}$ by Observation 13 and hence $\overline{S_{\infty}}=\mathbb{R}$ by Observation 16. But then $\overline{S_{0}}=-\overline{S_{\infty}}=\mathbb{R}$ by Observation $S$, and hence $\overline{S_{\infty}} \cap \overline{S_{0}} \cap S=S$. But $S \neq \emptyset$ since $S_{\infty} \cong S$, and hence this contradicts Observation 15. It follows that $E_{f}=\mathbb{1}$. Now $E\left(S_{f}\right)=\mathbb{1}$ by Observation 10, thus $E_{\omega}\left(S_{\infty}\right)=0$, and we may assume that $S_{\infty}=S_{0}=\emptyset$ by modifying $\psi_{1}, \psi_{2}$ on sets of $|\mu|+|v|$-measure zero.

It follows from Observation 8 that

$$
\begin{aligned}
& \left(\Delta^{1 / 2} A^{*} \Omega_{\omega}, \Delta^{1 / 2} P \Omega_{\omega}\right) \\
& \quad=\left(P^{*} \Omega_{\omega}, A \Omega_{\omega}\right) \\
& \quad=\left(\varphi\left(-H_{\omega}\right)^{1 / 2} A^{*} \Omega_{\omega}, \varphi\left(-H_{\omega}\right)^{1 / 2} P \Omega_{\omega}\right)
\end{aligned}
$$

for all $A, P \in \mathscr{M}_{\omega}$. As $\mathscr{M}_{\omega} \Omega$ is a core for $\Delta^{1 / 2}$, and $H_{\omega}$ and $\Delta^{1 / 2}$ commute strongly, it follows from this relation and a joint spectral representation of $H_{\omega}$ and $\Delta$ that

$$
\Delta=\varphi\left(-H_{\omega}\right) .
$$


It follows that

$\Sigma \cong \overline{\{(\log (\varphi(-p)), p) ; p \in \mathbb{R}\}}$.

Now, $\Sigma$ cannot have any isolated points by the reasoning used in Observation 12 , and $\Sigma$ is a closed subgroup of $\mathbb{R}^{2}$ by Observation 11 . As $\sigma\left(H_{\omega}\right)=\mathbb{R}$ it follows that $\Sigma$ must have one of the forms,

1. $\Sigma=\mathbb{R}^{2}$.

2. $\Sigma$ is an array of equidistant straight lines, not parallel with the $\log \Delta$-axis, one of which contains the origin.

3. $\Sigma$ is a straight line through the origin not coinciding with the $\log \Delta$-axis.

In Case 3, there exists a $\beta \in \mathbb{R}$ such that $\Sigma=\{(-\beta p, p) ; p \in \mathbb{R}\}$ and thus $\log \Delta$ $=-\beta H_{\omega}$, or

$$
\Delta=e^{-\beta H_{\omega}}
$$

but then $\omega$ is a $\tau$-KMS state at value $\beta$. Hence to complete the treatment of Case 1 , we must eliminate possibilities 1 and 2 above.

There are now two possibilities

Case 1a. $H_{A, A^{*}}=0$ for all $A \in \mathfrak{A}_{0}$. In this case

$$
\begin{aligned}
\omega\left(A A^{*}\right) & =F_{A, A^{*}}(0)+\omega(A) \omega\left(A^{*}\right) \\
& =G_{A, A^{*}}(0)+\omega(A) \omega\left(A^{*}\right) \\
& =\omega\left(A^{*} A\right)
\end{aligned}
$$

for all $A \in \mathfrak{A}_{0}$, and hence $\omega$ is a trace, i.e. $\omega$ is a $(\tau, 0)$-KMS state.

Case $1 b . \hat{H}_{A, A^{*}}\left(p_{0}\right) \neq 0$ for some $A \in \mathfrak{A}_{0}$ and $p_{0} \in \mathbb{R}$. Since $\hat{H}_{A, A^{*}}^{-}$is continuous, we may assume that $p_{0} \neq 0$ and that $\hat{H}_{A, A^{*}}(p) \neq 0$ for all $p \in\left(p_{0}-\varepsilon, p_{0}+\varepsilon\right)$, where $\varepsilon$ is some positive number. From Observation 6 and the relation

$$
d \mu_{A, A^{*}}(p)-d v_{A, A^{*}}(p)=\hat{H}_{A, A^{*}}(p) d p
$$

we deduce that

$$
\begin{aligned}
& (\varphi(p)-1) d v_{A, A^{*}}(p)=\hat{H}_{A, A^{*}}(p) d p \\
& \left(1-\varphi(p)^{-1}\right) d \mu_{A, A^{*}}(p)=\hat{H}_{A, A^{*}}(p) d p .
\end{aligned}
$$

As $\hat{H}_{A_{A} A^{*}}$ is a real function we have two possibilities: $\hat{H}_{A, A^{*}}\left(p_{0}\right) \gtrless 0$. If $\hat{H}_{A, A^{*}}\left(p_{0}\right)>0$, then $\hat{H}_{A, A^{*}}(p)>0$ for $p \in\left(p_{0}-\varepsilon, p_{0}+\varepsilon\right)$. We now deduce from the relation

$$
d v_{A, A^{*}}(p)=\hat{H}_{A, A^{*}}(p) d v(p)
$$

that $v$ is a positive measure on $\left(p_{0}-\varepsilon, p_{0}+\varepsilon\right)$ and

$$
(\varphi(p)-1) d v(p)=d p
$$

on this interval. It follows that $\varphi(p) \geqq 1$ for $p \in\left(p_{0}-\varepsilon, p_{0}+\varepsilon\right)$ except for a set of $|v|-$ measure zero. But $d \mu(p)=\varphi(p) d v(p)$ and so $\varphi(p) \geqq 1$ for $p \in\left(p_{0}-\varepsilon, p_{0}+\varepsilon\right)$ except for 
a set of $\mu$-measure, and hence spectral measure, zero. Thus we may choose $\varphi$ such that $\varphi(p) \geqq 1$ on $\left(p_{0}-\varepsilon, p_{0}+\varepsilon\right)$. But this means that the set

$$
\overline{\{(\log (\varphi(-p)), p) ; p \in \mathbb{R}\}}
$$

does not contain any point of the form $(d, p)$ where $d<0$ and $-p \in\left(p_{0}-\varepsilon, p_{0}+\varepsilon\right)$. As $\Sigma$ is contained in this set, this excludes possibilities 1 and 2 .

The case $\hat{H}_{A, A^{*}}\left(p_{0}\right)<0$ is treated by noting that $\hat{H}_{A^{*}, A}\left(-p_{0}\right)=-\hat{H}_{A, A^{*}}\left(p_{0}\right)$

Case 2. $E_{\omega}\left(S_{f}\right)$ is one-dimensional. If $E_{\omega}\left(S_{f}\right)=\mathbb{1}$ in this case, it follows that $\omega$ is a $\tau$ invariant character, and hence $\omega$ is a $\mathrm{KMS}$ state at all values $\beta \in \mathbb{R} \cup\{ \pm \infty\}$. If $E_{\omega}\left(S_{f}\right) \neq \mathbb{1}$, then $E_{\omega}\left(S_{\infty}\right)=\mathbb{1}-E_{\omega}\left(S_{f}\right) \neq 0$ and $S \neq \emptyset$ because $S_{\infty} \subseteq S$. We argue, ad absurdum, that $\overline{S_{\infty}} \neq \mathbb{R}$. If not, then $\overline{S_{0}}=-\overline{S_{\infty}}=\mathbb{R}$ and hence $\overline{S_{\infty}} \cap \overline{S_{0}} \cap S=S \neq \emptyset$ in contradiction with Observation 15 . Thus $\overline{S_{\infty}} \neq \mathbb{R}$, and by Observation 16

$$
\sigma\left(H_{\omega}\right) \subseteq \overline{S_{\infty}} \cup\{0\} \neq \mathbb{R} .
$$

But since $\sigma\left(H_{\omega}\right)$ has no isolated points it follows from [13] that $\sigma\left(H_{\omega}\right)$ is contained in one of the sets $\pm[0,+\infty\rangle$, and thus $\omega$ is a ground state or a ceiling state, i.e. $\omega$ is a $\mathrm{KMS}$ state at value $+\infty$ or $-\infty$.

By summarizing the results of the last two subsections, we obtain an almost completely satisfactory theory for the connection between stability and the KMS condition for $C^{*}$-dynamical systems $(\mathfrak{U}, \tau)$ which are $L^{1}\left(\mathfrak{U}_{0}\right)$-asymptotically abelian.

Assume that $\mathfrak{U}$ has an identity $\mathbb{1}$, and let $\omega$ be a $\tau$-stationary state on $\mathfrak{A}$. If $P=P^{*} \in \mathfrak{U}_{0}$, it follows that the Møller morphisms [16]

$$
\gamma_{ \pm}^{\lambda P}=\lim _{t \rightarrow \pm \infty} \tau_{-t}^{\lambda P} \tau_{t}
$$

exist strongly for $\lambda \in \mathbb{R}$. Furthermore one has the intertwining relations

$$
\gamma_{ \pm}^{\lambda P} \tau_{t}=\tau_{t}^{\lambda P} \gamma_{ \pm}^{\lambda P}
$$

and

$$
\lim _{\lambda \rightarrow 0} \gamma_{ \pm}^{\lambda P}(A)=A
$$

for all $A \in \mathfrak{A}$ by the estimate

$$
\left\|\gamma_{ \pm}^{\lambda P}(A)-A\right\| \leqq|\lambda| \int_{0}^{ \pm \infty} d|s|\left\|\left[P, \tau_{s}(A)\right]\right\|
$$

which is valid for $A \in \mathfrak{A}_{0}$.

Now, there exists a unique state $\omega^{\lambda P}$ on $\gamma_{-}^{\lambda P}(\mathfrak{A})$ satisfying

$$
\omega^{\lambda P}\left(\gamma_{-}^{\lambda P}(\mathrm{~A})\right)=\omega(A)
$$

and $\omega^{\lambda P}$ is $\tau^{\lambda P}$-stationary by the intertwining relations. But $\omega^{\lambda P}$ extends to a state of $\mathfrak{A}$, and applying an invariant mean to this extension composed with $\tau^{\lambda P}$ we obtain a state $\omega^{\lambda P}$ on $\mathfrak{U}$ such that the relation above remains valid, and 
1. $\omega^{\lambda P}$ is $\tau^{\lambda P}$-stationary

Next, it follows from the estimate

$$
\begin{aligned}
\left|\omega^{\lambda P}(A)-\omega(A)\right| & \leqq\left|\omega^{\lambda P}\left(A-\gamma_{-}^{\lambda P}(A)\right)\right| \\
& \leqq\left\|A-\gamma_{-}^{\lambda P}(A)\right\|
\end{aligned}
$$

that

2. $\lambda \mapsto \omega^{\lambda P}$ is continuous at $\lambda=0$ in the sense

$$
\lim _{\lambda \rightarrow 0} \omega^{\lambda P}(A)=\omega(A)
$$

for all $A \in \mathfrak{A}$.

Now, define a state $\omega_{+}^{\lambda P}$ on $\mathfrak{A}$ by

$$
\omega_{+}^{\lambda P}(A)=\omega^{\lambda P}\left(\gamma_{+}^{\lambda P}(A)\right) \text {. }
$$

From the relation

$$
\omega^{\lambda P}\left(\tau_{+}(A)\right)=\omega^{\lambda P}\left(\tau_{-t}^{\lambda P} \tau_{t}(A)\right)
$$

it follows that

3. The limits $\lim _{t \rightarrow \pm \infty} \omega^{\lambda P}\left(\tau_{t}(A)\right)$ exist for all $A \in \mathfrak{A}$, and

$$
\begin{aligned}
& \lim _{t \rightarrow-\infty} \omega^{\lambda P}\left(\tau_{t}(A)\right)=\omega(A) \\
& \lim _{t \rightarrow+\infty} \omega^{\lambda P}\left(\tau_{t}(A)\right)=\omega_{+}^{\lambda P}(A) .
\end{aligned}
$$

We call any family $\left\{\omega^{\lambda P} ; P=P^{*} \in \mathfrak{A}_{0},|\lambda|<\varepsilon_{P}\right\}$ of states satisfying the requirements 1,2 , and 3 above (including the existence of $\omega_{+}^{\lambda P}$ ) a family of perturbed states of $\omega$.

Corollary 7. Let $(\mathfrak{H}, \tau)$ be an $L^{1}\left(\mathfrak{U}_{0}\right)$-asymptotically abelian $C^{*}$-dynamical system, and assume that $\mathfrak{A}$ has an identity. Let $\omega$ be a $\tau$-stationary state of $\mathfrak{U}$, and let $\left\{\omega^{\lambda P} ; P=P^{*} \in \mathfrak{U}_{0},|\lambda|<\varepsilon_{P}\right\}$ be a family of perturbed states of $\omega$.

Consider the following conditions

$1_{\beta}$. $\omega$ is an extremal $\tau$-KMS state at value $\beta$.

2. a) $\omega$ has the three point cluster property

$$
\begin{aligned}
& \lim _{\substack{i \neq j \\
i \neq j}} \lim _{t_{i}-t_{j} \mid \rightarrow \infty} \omega\left(\tau_{t_{1}}\left(A_{1}\right) \tau_{t_{2}}\left(A_{2}\right) \tau_{t_{3}}\left(A_{3}\right)\right) \\
& =\omega\left(A_{1}\right) \omega\left(A_{2}\right) \omega\left(A_{3}\right) .
\end{aligned}
$$

b) $\omega$ satisfies the stability property

$$
\lim _{\lambda \rightarrow 0}\left|\omega_{+}^{\lambda P}(A)-\omega(A)\right| / \lambda=0
$$

for all $A \in \mathfrak{U}$. 
It follows that 2 implies $1_{\beta}$ for some $\beta \in \mathbb{R} \cup\{ \pm \infty\}$. Conversely $1_{\beta}$ for $\beta \in(\mathbb{R} \cup\{ \pm \infty\}) \backslash\{0\}$ implies 2 , and $1_{\beta}$ for $\beta=0$ implies 2 when $\left.2 a\right)$ is replaced by the weaker cluster property

$$
M \omega(A \tau(B))=\omega(A) \omega(B)
$$

for all $A, B \in \mathfrak{A}$, and any invariant mean $M$ on $\mathbb{R}$.

In particular, $1_{\beta}$ for some $\beta \in \mathbb{R} \cup\{ \pm \infty\}$ and 2 are equivalent if $\omega$ is a factor state or if $\mathfrak{U}$ has a unique trace state.

Furthermore the family of perturbations $\left\{\omega^{\lambda P}\right\}$ can be chosen such that $\omega_{+}^{\lambda P}=\omega$ in the following cases

A) If 1 . holds with $\beta \in \mathbb{R}$.

B) If 1 . holds with $\beta=+\infty$ and there exists an $\varepsilon>0$ such that

$\sigma\left(H_{\omega}\right) \subseteq\{0\} \cup[\varepsilon,+\infty\rangle$.

C) If 1 . holds with $\beta=+\infty$, and ( $\mathfrak{H}, \tau)$ has a unique ground state.

In these cases $\omega^{\lambda P}$ can even be taken to be a $\tau^{\lambda P}$-KMS state at value $\beta$.

Proof. We first show that the stability condition $2 \mathrm{~b}$ ) is equivalent to the by now familiar condition

$$
\int_{-\infty}^{\infty} d t \omega\left(\left[P, \tau_{t}(A)\right]\right)=0
$$

for all $A, P \in \mathfrak{A}_{0}$. But this is a consequence of the relation

$$
\tau_{-t}^{\lambda P} \tau_{t}(A)=A-i \lambda \int_{0}^{t} d s \tau_{-s}^{\lambda P}\left(\left[P, \tau_{s}(A)\right]\right)
$$

which gives

$$
\begin{aligned}
\omega(A) & =\omega^{\lambda P}\left(\gamma_{-}^{\lambda P}(A)\right) \\
& =\omega^{\lambda P}(A)-i \lambda \int_{0}^{-\infty} d s \omega^{\lambda P}\left(\left[P, \tau_{s}(A)\right]\right), \\
\omega_{+}^{\lambda P}(A) & =\omega^{\lambda P}\left(\gamma_{+}^{\lambda P}(A)\right) \\
& =\omega^{\lambda P}(A)-i \lambda \int_{0}^{\infty} d s \omega^{\lambda P}\left(\left[P, \tau_{s}(A)\right]\right),
\end{aligned}
$$

and hence

$$
\begin{aligned}
& \left(\omega_{+}^{\lambda P}(A)-\omega(A)\right) / \lambda \\
& \quad=-i \int_{-\infty}^{\infty} d s \omega^{\lambda P}\left(\left[P, \tau_{s}(A)\right]\right) .
\end{aligned}
$$

The Lebesgue dominated convergence theorem and requirement 2 on the family $\left\{\omega^{\lambda P}\right\}$ now immediately imply that the two stability conditions are equivalent.

Thus it follows from Theorem 6 that 2 implies $1_{\beta}$ for some $\beta \in \mathbb{R} \cup\{ \pm \infty\}$. But $1_{\beta}$ for $\beta \in \mathbb{R} \cup\{ \pm \infty\}$ implies $(*)$ by Theorem 4 . 
Now, $1_{\beta}$ for $\beta \in \mathbb{R} \backslash\{0\}$ implies that $\omega$ is a factor state and if $1_{\beta}$ is true for $\beta \in\{ \pm \infty\}$ then $\omega$ is pure. Thus $\omega$ is a factor state in both cases, and it follows from the asymptotic abelianness that

$$
\begin{aligned}
& \lim _{\substack{\inf _{\imath \neq j}\left|t_{i}-t_{j}\right| \rightarrow \infty\\
}} \omega\left(\tau_{t_{1}}\left(A_{1}\right) \ldots \tau_{t_{n}}\left(A_{n}\right)\right) \\
& =\omega\left(A_{1}\right) \ldots \omega\left(A_{n}\right)
\end{aligned}
$$

for all $n \in \mathbb{Z}_{+}$and all $A_{i} \in \mathfrak{A},[7]$.

If $1_{\beta}$ holds for $\beta=0$, i.e. $\omega$ is an extremal invariant trace, then $\omega$ is an extremal invariant state by asymptotic abelianness, and thus

$$
M \omega(A \tau(B))=\omega(A) \omega(B) \text {. }
$$

If $\omega$ is assumed to be a factor state, one derives $n$-point clustering as above, and hence 2 and $1_{\beta}$, for some $\beta \in \mathbb{R} \cup\{ \pm \infty\}$, are completely equivalent.

But if $\mathfrak{U}$ has a unique trace state then every extremal $(\tau, \beta)$-KMS state $\omega$ must be a factor state.

(If $\beta=0$ then $\omega$ is the unique trace and is automatically a factor state.) The equivalence and 2 and $1_{\beta}$, for some $\beta \in \mathbb{R} \cup\{ \pm \infty\}$, follows once again.

The last statement of the Corollary follows from combining the results of [2] and [16] for $\beta \in \mathbb{R}$ and the rest follows from Proposition 5 and the preceding remarks.

\section{References}

1. Aizenman,M., Gallavotti,G., Goldstein,S., Lebowitz,J.L.: Stability and equilibrium states of infinite classical systems. Commun. math. Phys. 48, 1-14 (1976)

2. Araki,H.: Relative Hamiltonian for faithful normal states of a von Neumann algebra. Publ. Res. Inst. Math. Sci., Kyoto 9, 165-209 (1973)

3. Araki,H.: Positive cone, Radon-Nikodym theorems, relative Hamiltonian and the Gibbs condition in statistical mechanics. An application of the Tomita-Takesaki theory, in $C^{*}$-algebras and their applications to statistical mechanics and quantum field theory. Bologna: Editrice Compositori 1975

4. Arveson, W.: On groups of automorphisms of operator algebras. J. Funct. Anal. 15, 217-243 (1974)

5. Borchers, H.J.: Energy and momentum as observables in quantum field theory. Commun. math. Phys. 2, 49-54 (1966)

6. Bratteli,O., Kastler,D.: Relaxing the clustering condition in the derivation of the KMS property. Commun. math. Phys. 46, 37-42 (1976)

7. Bratteli, O., Robinson,D.W.: Operator algebras and quantum statistical mechanics. (to appear)

8. Connes, A.: Une classification des facteurs de type III. Ann. Sc. Ec. Norm. Sup. 6, 133-252 (1973)

9. Dixmier,J.: Les $C^{*}$-algèbres et leurs représentations. Paris: Gauthier-Villars 1964

10. Haag, R., Kastler,D., Trych-Pohlmeyer,E.B.: Stability and equilibrium states. Commun. math. Phys. 38, 173-193 (1974)

11. Haag, R., Trych-Pohlmeyer,E. B.: Stability properties of equilibrium states. Commun. math. Phys. 56, 213-224 (1977)

12. Hoekman,F.: On stability and symmetries in quantum statistical mechanics. Ph. D. Thesis, Groningen (1977)

13. Kastler,D.: Equilibrium states of matter and operator algebras. Symp. Math. 20, 49-107 (1976)

14. Pulvirenti,M.: Stability, equilibrium and KMS for an infinite classical system. J. Math. Phys. 18, 2099-2103 (1977) 
15. Pusz,W., Woronowicz,S.L.: Passive states and KMS states for general quantum systems. Commun. math. Phys. 58, 273-290 (1978)

16. Robinson,D.W.: Return to equilibrium. Commun. math. Phys. 31, 171-189 (1973)

17. Robinson,D.W.: $C^{*}$-algebras and quantum statistical mechanics, in $C^{*}$-algebras and their applications to statistical mechanics and quantum field theory. Bologna: Editrice Composition 1975

18. Sewell,G.L.: KMS conditions and local thermodynamic stability of quantum lattice systems. II. Commun. math. Phys. 55, 53-61 (1977)

Communicated by E. Lieb

Received March 6, 1978 